\title{
Time-domain properties of electromagnetic signals in a dynamical axion background
}

\author{
Peter Adshead, ${ }^{*}$ Patrick Draper, ${ }^{\dagger}$ and Benjamin Lillard $\odot^{\ddagger}$ \\ Illinois Center for Advanced Studies of the Universe and Department of Physics, \\ University of Illinois at Urbana-Champaign, Urbana, Illinois 61801, USA
}

(Received 14 August 2020; accepted 20 November 2020; published 7 December 2020)

\begin{abstract}
Electromagnetic waves in a dynamical axion background exhibit superluminal group velocities at high frequencies and instabilities at low frequencies, altering how photons propagate through space. Local disturbances propagate causally, but unlike in ordinary Maxwell theory, propagation occurs inside as well as on the light cone. For the unstable modes, the energy density in the electromagnetic field grows exponentially along timelike displacements. In this paper we derive retarded Green functions in axion electrodynamics in various limits and study the time-domain properties of propagating signals.
\end{abstract}

DOI: 10.1103/PhysRevD.102.123011

\section{INTRODUCTION}

Axion models provide some of the most well-motivated extensions to the Standard Model, providing a mechanism to resolve the strong $C P$ problem and a class of dark matter candidates. Through its coupling to gluons, the vacuum expectation value of the axion field cancels the $\bar{\theta}$ parameter of quantum chromodynamics (QCD) and restores $C P$ symmetry $[1,2]$, explaining the surprisingly small experimentally measured value, $|\bar{\theta}|<6 \times 10^{-11}[3,4]$. Through the misalignment mechanism [5-7], axions can also be produced in the early Universe in sufficient abundance to comprise most or all of the dark matter.

Many of the most stringent constraints on axion models utilize a coupling between the axion and the Standard Model electric and magnetic fields,

$$
\mathcal{L} \supset-\frac{1}{8} g_{a \gamma \gamma} a \epsilon^{\mu \nu \rho \sigma} F_{\mu \nu} F_{\rho \sigma}=g_{a \gamma \gamma} a \mathbf{E} \cdot \mathbf{B} .
$$

This coupling enables axion production through the Primakoff process [8,9], axion decay to photons, and axion-photon interconversion in the presence of electromagnetic fields. As such it can be used either to detect or to produce axions in the laboratory. Stellar observations [10-12] constrain $g_{\text {ary }}<10^{-10} \mathrm{GeV}^{-1}$ for a wide range of

\footnotetext{
adshead@illinois.edu

†pdraper@illinois.edu

blillard@illinois.edu
}

Published by the American Physical Society under the terms of the Creative Commons Attribution 4.0 International license. Further distribution of this work must maintain attribution to the author(s) and the published article's title, journal citation, and DOI. Funded by SCOAP ${ }^{3}$. axion masses, and additional constraints set by the power spectra of bright $\mathrm{x}$-ray point sources provide a more stringent limit of $g_{\text {ary }} \lesssim 10^{-12} \mathrm{GeV}^{-1}$ for light axions of mass $m_{a} \lesssim 10^{-12} \mathrm{eV}$ [13-15]. At large occupation numbers and de Broglie wavelengths, axion dark matter behaves as a classical, oscillating background field that induces small time-dependent perturbations to electrodynamics, which can be probed with a variety of different sensitive experimental techniques [9,16-21].

The coupling in Eq. (1) also affects the propagation of classical electromagnetic radiation [22-27]. Electromagnetic plane waves traveling through an axion background acquire modified phase velocities for left- and right-polarizations, an effect which may be observable in interferometers [28-30], atomic clocks [31] or astrophysical sources [32], for some ranges of axion masses and couplings. If axion domain walls form in the early Universe, the modified dispersion relations provide a new mechanism for energy dissipation via photon reflection [33]. Furthermore, low frequency modes exhibit tachyonic instabilities, while at high frequencies, group velocities for both polarizations are superluminal.

Despite the presence of plane wave solutions with superluminal group velocities, axion electrodynamics is a causal theory: local disturbances do not propagate outside the light cone. This was first shown long ago in the case of tachyonic scalar field theory by Aharonov, Komar, and Susskind [34]. Here we show that the same mechanism is at work in axion electrodynamics, with consequences that include the exponential growth of local disturbances.

In this paper we calculate the classical electromagnetic retarded Green function in a coherent, dynamical axion background in several disparate regimes of axion parameter space. Our results are organized based on the hierarchical ordering of three different scales: 
(i) $m_{a}$, the axion mass;

(ii) $\mu_{0} \equiv \frac{1}{2} g_{a \gamma \gamma} \sqrt{\rho_{a}}$, a mass scale that determines the rate of exponential growth of the electromagnetic fields, based on the axion density $\rho_{a}$; and

(iii) $T^{-1}$ and $L^{-1}$, the inverses of the characteristic propagation time and distance $T \sim L$ of a signal.

Rather than focusing only on those axion models that provide a natural solution to the strong $C P$ problem, we consider the broader realm of axionlike particles (ALPs), where the axion mass $m_{a}$ and decay constant $f_{a}$ are not required to satisfy $m_{a} f_{a} \sim m_{\pi} f_{\pi}$, and the value of $g_{a \gamma \gamma}$ is not determined by $m_{a}$. These axions can still provide a wide range of dark matter candidates (see, e.g., [35-37]). For the lowest-mass "fuzzy dark matter" candidates, generic constraints on ultralight scalars from Lyman- $\alpha$ data [38-41] impose a lower bound on the axion mass of $m_{a} \gtrsim 2 \times 10^{-21} \mathrm{eV}$, though in the "large misalignment" regime of Ref. [42] the Lyman- $\alpha$ bound is altered by the effect of ALP self-interactions. Our analysis in this paper encompasses the nearly 20 decades of ALP parameter space above this bound, where the axion is still light enough that it can be treated as a coherently oscillating background field.

In terms of the axion virial velocity $v$, the mass sets an upper bound on the characteristic $L$ and $T, L \lesssim\left(m_{a} v\right)^{-1}$ and $T \lesssim\left(m_{a} v^{2}\right)^{-1}$, after which any analysis must incorporate the effects of decoherence. In the example of fuzzy dark matter with $m_{a} \approx 2 \times 10^{-21} \mathrm{eV}$, and taking $v \sim 10^{-3}$ as the ALP virial velocity, the coherence length and time are, respectively, $L_{c} \lesssim 10^{17} \mathrm{~m} \approx 3 \mathrm{pc}$ and $T_{c} \lesssim 3 \times$ $10^{11} \mathrm{~s} \approx 10^{4} \mathrm{yr}$. On the other extreme, for $m_{a}$ much larger than $10^{-4} \mathrm{eV}$, even a tabletop experiment will encounter significant decoherence. In the presence of cold axion streams, where some population of the dark matter has a significantly smaller virial velocity, the relevant coherence time can be much longer. Our analysis is focused on the nonrelativistic limit, neglecting these decoherence effects and ignoring spatial gradients in the axion field.

We begin in Sec. II with the simplest analysis, the $m_{a} \ll \mu_{0}, 1 / T$ limit. In this case the value of the axion field changes at an approximately constant rate, $\partial_{t} a(x, t) \approx$ const, and we find an analytic solution to the Green function valid for all values of $\mu_{0} T$. This Green function exhibits exponential growth inside the light cone of the disturbance when $\mu_{0} T \gtrsim 1$. Despite the potentially catastrophic consequences of this unbounded growth, the dilute density of dark matter and experimental constraints on the axion-photon coupling ensure that the timescales for the genuinely exponential phase of the growth are outside the reach of all but the lightest ALP candidates, unless the local ALP density $\rho_{a}$ is enhanced by several orders of magnitude above $0.4 \mathrm{GeV} / \mathrm{cm}^{3}$. In Sec. II C we highlight some of the curious and potentially detectable perturbations to classical electrodynamics induced by the axion background.
For almost all allowed values of $g_{a \gamma \gamma}$ and $\rho_{a}$, the hierarchy $\mu_{0} \ll m_{a}$ is more realistic, and we explore this limit in Sec. III. In the case of the oscillating background axion field it is no longer possible to derive an exact analytic expression for the Green function using the methods of Sec. II. Instead, we construct perturbative expansions for the $\mu_{0} T \ll 1$ and $\mu_{0} T \gg 1$ limits by expressing the Green function as a continued fraction. When the frequency support of the radiation includes $\omega \approx \frac{1}{2} m_{a}$, a narrow resonance induces exponential growth for large $T$. In Sec. III A we calculate the dominant part of the Green function in the $\mu_{0} T \gg 1$ limit. In this late-time limit the resonant enhancement dwarfs the contribution from frequencies $\omega \neq \frac{1}{2} m_{a}$. This resonant emission has been previously studied in [43,44], although the resonant band is so narrow that dispersion effects and gravitational redshifting may completely prevent the exponential growth [45]. For the nonresonant limit $\mu_{0} T \ll 1$, and for electromagnetic signals which do not include support near the resonant frequency $\omega \approx \frac{1}{2} m_{a}$, Sec. III B provides a continued fraction expression for the Green function that is valid to arbitrary order in $g_{\text {ary }}$. In Secs. II C and III C, we provide numeric examples to illustrate the behavior of signals in various corners of ALP parameter space, and to verify our analytic expressions.

The primary results of this paper are collected in Eqs. (77), (78) and (79) in Sec. IV. Despite the significant differences between the two limits, the Green functions of Secs. II and III both exhibit the novel inside-the-light-cone propagation and exponential growth in certain modes.

\section{A. Axion electrodynamics}

In terms of $\theta\left(x^{\mu}\right)$, the local value of the effective $C P$ violation induced by the axion background, the Lagrangian for electrodynamics includes the interactions

$$
\mathcal{L}=-\frac{1}{4} F_{\mu \nu} F^{\mu \nu}-A_{\mu} J^{\mu}+\frac{\theta}{8} \epsilon^{\mu \nu \rho \sigma} F_{\mu \nu} F_{\rho \sigma},
$$

where $F_{\mu \nu}$ is the electromagnetic field strength tensor, $A_{\mu}$ and $J^{\mu}$ are the vector potential and 4-current, and $\theta\left(x^{\mu}\right)$ is related to the value of the axion field via

$$
\theta\left(x^{\mu}\right) \equiv g_{a \gamma \gamma} a\left(x^{\mu}\right) .
$$

In Lorenz $\partial_{\alpha} A^{\alpha}=0$ gauge, the equations of motion for $A_{\mu}$ reduce to

$$
\partial^{2} A^{\mu}-\epsilon^{\mu \nu \rho \sigma}\left(\partial_{\nu} \theta\right)\left(\partial_{\rho} A_{\sigma}\right)=J^{\mu},
$$

which depends explicitly on the derivatives of $\theta\left(x^{\mu}\right)$ rather than $\theta$ itself. Taking the external source to be neutral and transverse, $J^{0}=0$ and $\nabla \cdot J=0$, and neglecting any spatial gradients in the background axion field, $|\nabla a| \ll|\dot{a}|$, 
the equations of motion for the scalar and vector potentials decouple, ${ }^{1}$

$$
\partial^{2} \Phi=0, \quad \partial^{2} \mathbf{A}+\dot{\theta} \nabla \times \mathbf{A}=\mathbf{J} .
$$

For a typical model of ALP dark matter, $\dot{\theta}(t)$ is given by

$$
\theta(t) \approx \theta_{0} \cos \left(m_{a} t\right), \quad \theta_{0}=g_{a \gamma \gamma} \frac{\sqrt{\rho_{a}}}{m_{a}}, \quad \dot{\theta}_{0}=m_{a} \theta_{0}
$$

where the value of $\theta_{0}$ is set by the local axion dark matter density, $\rho_{a} \sim(0.042 \mathrm{eV})^{4}$, and where

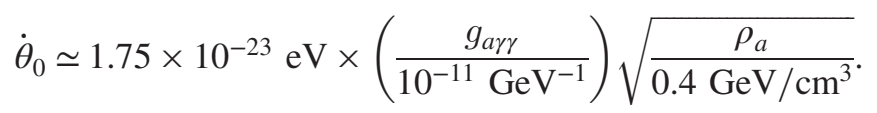

For future reference, we note that $1.75 \times 10^{-23} \mathrm{eV} \simeq$ $2.66 \times 10^{-8} \mathrm{~Hz} \simeq 0.84 \mathrm{yr}^{-1}$.

\section{GREEN FUNCTIONS FOR THE STEADY-STATE BACKGROUND}

For timescales that are short compared to the period of the axion oscillation, $m_{a} t \ll 1$ and $m_{a} \lesssim \dot{\theta}$, it is appropriate and instructive to consider the approximation $\partial_{t}^{2} \theta \approx 0$, where $\dot{\theta}(t)$ assumes a nearly constant value $-\dot{\theta}_{0} \leq \dot{\theta}(t) \leq \dot{\theta}_{0}$. In this case the differential equation for A can be solved using a Fourier transform,

$$
\mathbf{A}(t, \mathbf{x})=\left(\frac{1}{2 \pi}\right)^{4} \int d \omega d^{3} \mathbf{k} \tilde{\mathbf{A}}(\omega, \mathbf{k}) e^{i(\omega t-\mathbf{k} \cdot \mathbf{x})},
$$

so that the differential equation for $\mathbf{A}$ becomes a set of algebraic equations for $\tilde{A}_{i}$. For planar waves propagating in the $\hat{z}$ direction, $\mathbf{k}=k \hat{z}$, the polarization basis $\mathbf{A}=A_{+} \hat{\epsilon}_{+}+$ $A_{-} \hat{\epsilon}_{-}+A_{z} \hat{z}$ with $\hat{\epsilon}_{ \pm}=\frac{1}{\sqrt{2}}(\hat{x} \pm i \hat{y})$ diagonalizes the equations of motion, with the result

$$
\tilde{A}_{ \pm}\left[k^{2}-\omega^{2} \pm \dot{\theta} k\right]=\tilde{j}_{ \pm}(\mathbf{k}), \quad \tilde{A}_{z}\left[k^{2}-\omega^{2}\right]=0,
$$

where $\tilde{j}_{ \pm}(k)$ is the Fourier transform of the transverse source $\left(\hat{\epsilon}_{ \pm} \cdot \mathbf{J}\right)$ in the polarization basis. Circularly polarized plane waves propagate with the dispersion relations

$$
\omega_{ \pm}^{2}=k^{2} \pm k \dot{\theta},
$$

producing subluminal or superluminal phase velocities depending on the sign of $\dot{\theta}$ and the polarization of the radiation.

\footnotetext{
${ }^{1}$ The equations of motion with $\nabla a \neq 0$ are discussed in e.g., $[23,46]$.
}

The group velocities for both modes are superluminal, for both positive and negative $\dot{\theta}$,

$$
\frac{d \omega_{ \pm}}{d k}=\frac{k \pm \frac{1}{2} \dot{\theta}}{\sqrt{k^{2} \pm k \dot{\theta}}} \geq 1,
$$

with $d \omega_{ \pm} / d k=1$ only for $\dot{\theta}=0$. The effect appears at quadratic order in $\dot{\theta} / k$,

$$
\frac{d \omega_{ \pm}}{d k}=1+\frac{1}{8} \frac{\dot{\theta}^{2}}{k^{2}} \mp \frac{1}{8} \frac{\dot{\theta}^{3}}{k^{3}}+\ldots,
$$

so that for $\dot{\theta} \ll k$ the modification of the group velocity is subdominant to the $\mathcal{O}(\dot{\theta} / k)$ change in the phase velocity,

$$
\frac{\omega_{ \pm}}{k}=\sqrt{1 \pm \frac{\dot{\theta}}{k}}=1 \pm \frac{1}{2} \frac{\dot{\theta}}{k}-\frac{1}{8} \frac{\dot{\theta}^{2}}{k^{2}} \pm \frac{1}{16} \frac{\dot{\theta}^{3}}{k^{3}}+\ldots
$$

We demonstrate below that the retarded Green function vanishes outside the light cone, preserving causality despite the presence of superluminal group velocities. As a necessary consequence, disturbances in the field induced by local sources grow exponentially in timelike directions. To leading order in $\dot{\theta}$, the phase velocities alternate about a central value $\omega_{ \pm} / k=1$ based on the polarization of the light and the sign of $\dot{\theta}$. After multiple periods of the axion oscillation, the perturbations to the phase velocity tend to cancel each other. On the other hand, the group velocity is superluminal for both positive and negative $\dot{\theta}$, so the exponential growth is not ameliorated by any periods of exponential decay when the sign of $\dot{\theta}$ changes. The effects from the modified group velocities should grow over time.

With the approximation that $\dot{\theta}(t)$ is nearly constant, the two-dimensional Green function can be obtained analytically to all orders in $\dot{\theta}$. Experimental constraints on $g_{\text {ary }}<10^{-10} \mathrm{GeV}^{-1}$ and $m_{a}$ exclude the $m_{a} \ll \dot{\theta}$ possibility unless the local axion density is significantly enhanced, $\rho_{a} \gg \mathcal{O}\left(\mathrm{GeV} / \mathrm{cm}^{3}\right)$, so the results in this section are directly applicable primarily to situations involving to dense clumps of ultralight axions. When we calculate the Green function for the more broadly relevant $m_{a} \gg \dot{\theta}$ hierarchy of scales in Sec. III, the steady-state case with constant $\dot{\theta}$ also provides a helpful consistency check in the limit where the exponential growth becomes important.

\section{A. Green function solution in two dimensions}

The Green function can be calculated analytically for the simplified case of plane waves $\mathbf{k}=k \hat{z}$ with a spatially homogenous $(\nabla \theta=0)$, steady-state $\left(\partial_{t}^{2} \theta=0\right)$ axion background. Imposing translational symmetry in $x$ and $y$ effectively reduces the system from $(3+1)$ dimensions to $(1+1)$. The $\tilde{A}_{ \pm}$equation of motion in Eq. (9) admits a Green function solution $g_{ \pm}(z, t)$ of the form 


$$
A_{ \pm}=\int d t_{0} d z_{0} j_{ \pm}\left(t_{0}, z_{0}\right) g_{ \pm}\left(t-t_{0}, z-z_{0}\right)
$$

where

$$
\left(\partial_{t}^{2}-\partial_{z}^{2} \pm i \dot{\theta} \partial_{z}\right) g_{ \pm}\left(t-t_{0}, z-z_{0}\right)=\delta\left(z-z_{0}\right) \delta\left(t-t_{0}\right) .
$$

In this section it is convenient to fold a factor of $1 / 2$ into the definition of $\dot{\theta}$,

$$
\mu(t)=\frac{\dot{\theta}(t)}{2},
$$

where $\mu$ determines the rate of exponential growth, as we show below.

Defining a related Green function $G_{0}$,

$$
g_{ \pm}\left(t\left|t_{0}, z\right| z_{0}\right)=e^{ \pm i \mu z} G_{0}\left(t\left|t_{0}, z\right| z_{0}\right),
$$

Eq. (15) can be simplified to

$$
\left(\partial_{t}^{2}-\partial_{z}^{2}-\mu^{2}\right) G_{0}(t, z)=e^{\mp i \mu z} \delta(z) \delta(t),
$$

so that $\mu^{2}$ acts as an effective tachyonic mass for the scalarlike Green function $G_{0}$.

Applying the Fourier transform and integrating both sides of Eq. (18) produces the integral form of the Green function,

$$
G_{\epsilon}=\int \frac{d \omega d k}{(2 \pi)^{2}} \frac{e^{-i(\omega t-k z)}}{k^{2}-(\omega+i \varepsilon)^{2}-\mu^{2}},
$$

where $\varepsilon>0$ indicates that the contour in the complex $\omega$ plane should correspond to the retarded Green function, which vanishes for $t<0$. For $k^{2}>\mu^{2}$ the $\varepsilon \rightarrow 0^{+}$limit can be recovered easily. However, for $k^{2}<\mu^{2}$ one of the poles in $\omega$ is located above the real axis, at

$$
\omega=-i \varepsilon \pm i \sqrt{\mu^{2}-k^{2}} .
$$

To recover the retarded Green function, the contour in $\omega$ should pass above both poles, with $\varepsilon \rightarrow \mu^{+}$on the imaginary axis.

The $\int d \omega$ integral can be completed using the residue theorem,

$$
G_{\epsilon}=\Theta(t) \frac{-2 \pi i e^{-\varepsilon}}{(2 \pi)^{2}} \int \frac{d k e^{i k z}}{2 \omega_{0}}\left(e^{i \omega_{0} t}-e^{-i \omega_{0} t}\right)
$$

where $\omega_{0}=\sqrt{k^{2}-\mu^{2}}=i \sqrt{\mu^{2}-k^{2}}$ and $\Theta(t)$ is the step function. The integral is simplified by a coordinate substitution $k \rightarrow \varphi$,

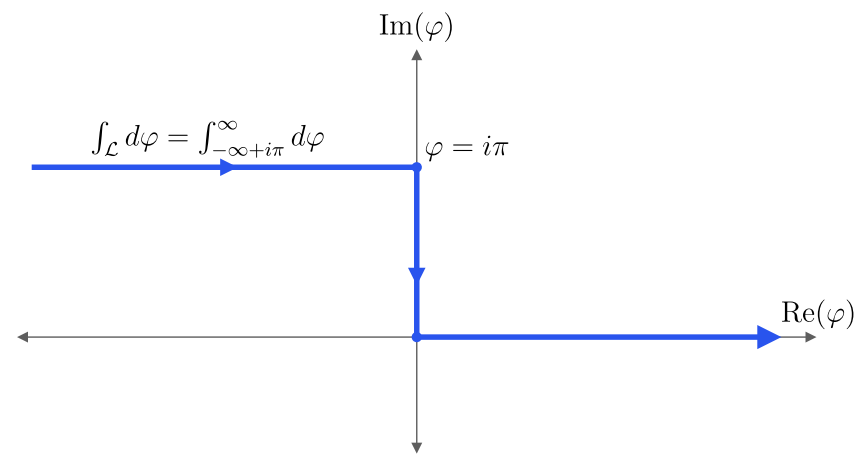

FIG. 1. The contour $\mathcal{L}$ that allows Eq. (24) to be written in terms of Hankel functions. The shorthand notation for this contour is $\int_{-\infty+i \pi}^{\infty} d \varphi$, indicating that the contour approaches the imaginary axis from negative real $\infty$ with a constant imaginary component of $+i \pi$.

$$
k=\mu \cosh \varphi, \quad \omega_{0}=\mu \sinh \varphi,
$$

where the contour $\mathcal{L}$ in the complex $\varphi$ plane is shown in Fig. 1, and allows $\cosh \varphi$ to vary smoothly from $-\infty$ to $\infty$ with $\operatorname{Im}(\cosh \varphi)=0$. By mapping the coordinates $(t, z)$ to $(\eta, \lambda)$ via

$\mu t=\sqrt{\lambda} \cosh \eta, \quad \mu z=\sqrt{\lambda} \sinh \eta, \quad t^{2}-z^{2}=\lambda / \mu^{2}$,

for $\lambda \geq 0$, the integral can be written in terms of just $\lambda$ and $\varphi \pm \eta$ :

$$
\begin{aligned}
G_{0}(\lambda \geq 0) & =\frac{\Theta(t)}{4 \pi i} \int_{\mathcal{L}} d \varphi\left(e^{i \sqrt{\lambda} \sinh (\varphi+\eta)}-e^{-i \sqrt{\lambda} \sinh (\varphi-\eta)}\right), \\
& =\frac{\Theta(t)}{4 \pi i} \int_{-\infty+i \pi}^{\infty} d \varphi^{\prime}\left(e^{i \sqrt{\lambda} \sinh \varphi^{\prime}}-e^{-i \sqrt{\lambda} \sinh \varphi^{\prime}}\right) .
\end{aligned}
$$

Here the notation $\int_{-\infty+i \pi}^{\infty} d \varphi$ indicates the imaginary offset for $\operatorname{Re}(\varphi)<0$ shown in Fig. 1. In order to make this last simplification, removing the $\eta$ dependence completely, note that the integrand has no poles for finite $\varphi^{\prime}$, so that the contours $\mathcal{L}_{ \pm}$from the coordinate substitutions $\varphi_{ \pm}=\eta \pm \varphi$ can be shifted horizontally to compensate for $\eta$.

Outside the light cone, for spacelike displacements $z^{2}>t^{2}$, the coordinate transformation Eq. (23) is replaced by the alternative

$\mu z=\bar{z} \cosh \eta, \quad \mu t=\bar{z} \sinh \eta, \quad z^{2}-t^{2}=\bar{z}^{2}=-\lambda / \mu^{2}$.

In this case, with $\lambda<0$, the two contributions to the integral cancel each other, 
$G_{0}(\lambda<0)=\frac{\Theta(t)}{4 \pi i} \int_{\mathcal{L}} d \varphi\left(e^{i \bar{z} \cosh (\varphi+\eta)}-e^{i \bar{z} \cosh (\varphi-\eta)}\right)=0$,

and so the retarded Green function vanishes outside the light cone.

Using this contour notation, and the integral definition of the Hankel functions

$$
H_{\nu}^{(1,2)}(z)= \pm \frac{1}{\pi i} \int_{-\infty}^{+\infty \pm i \pi} d u e^{z \sinh u-\nu u}
$$

$G_{0}$ can be written as

$G_{0}(t, z)=\frac{\Theta(t) \Theta(\lambda) i}{4 \pi}\left(\frac{\pi}{i} H_{0}^{(2)}(i \sqrt{\lambda})-\frac{\pi}{i} H_{0}^{(2)}(-i \sqrt{\lambda})\right)$.

Equation (28) simplifies for integer values of $\nu$ to recover an expression in terms of the modified Bessel function of the first kind, $I_{\nu}(z)$ :

$$
G_{0}(t, z)=\frac{\Theta(t) \Theta(\lambda)}{2} I_{0}(\sqrt{\lambda}) .
$$

Finally, we obtain

$$
g_{ \pm}(t, z)=e^{ \pm i \mu z} \frac{\Theta(t) \Theta\left(t^{2}-z^{2}\right)}{2} I_{0}\left(\sqrt{\mu^{2}\left(t^{2}-z^{2}\right.}\right) .
$$

As anticipated by the discussion of tachyonic scalar theories in Ref. [34], the Bessel function $I_{0}(\sqrt{\lambda})$ grows exponentially with timelike $\lambda \propto t^{2}-z^{2}$. In the $\lambda \gg 1$ limit it approaches

$$
\lim _{\lambda \rightarrow \infty} I_{0}(\sqrt{\lambda})=\frac{\lambda^{-1 / 4} e^{\sqrt{\lambda}}}{\sqrt{2 \pi}}\left(1+\frac{1}{8 \sqrt{\lambda}}+\mathcal{O}\left(\lambda^{-1}\right)\right) .
$$

The retarded Green function vanishes outside the light cone, $t^{2}<z^{2}$, and it explicitly satisfies the equations of motion

$$
\begin{aligned}
& \int d t_{0} d z_{0} j_{ \pm}\left(t_{0}, z_{0}\right)\left(\partial_{t}^{2}-\partial_{z}^{2} \pm i \dot{\theta} \partial_{z}\right) g_{ \pm}\left(t-t_{0}, z-z_{0}\right) \\
& =j_{ \pm}(t, z) .
\end{aligned}
$$

From the solution for $A_{ \pm}$one can derive the corresponding Green functions for the $\mathbf{E}$ and $\mathbf{B}$ fields,

$E_{x}=-\partial_{t} A_{x}, \quad E_{y}=\partial_{t} A_{y}, \quad B_{x}=-\partial_{z} A_{y}, \quad B_{y}=\partial_{z} A_{x}$,

with $E_{z}=B_{z}=0$.
In many of the simplest cases of interest, including idealized axion interferometers and astrophysical sources, the photon source is localized in space and produces a signal $s(\tau, z)=\delta(z) s(\tau)$ or alternatively $s(\tau, z)=s(\tau-z)$ that varies in time and propagates in the forward direction, $z \geq 0$. With this choice, the $\mathbf{E}$ and $\mathbf{B}$ fields can be found from the $\Theta(z)$ components of the derivatives of $A_{ \pm}$, and the familiar Standard Model limit $\mu \rightarrow 0$ is recovered by

$$
\begin{gathered}
\partial_{t} A_{ \pm}^{\mu=0}(t, z)=-\Theta(z) \frac{1}{2} j_{ \pm}(t-z), \\
\partial_{z} A_{ \pm}^{\mu=0}(t, z)=\Theta(z) \frac{1}{2} j_{ \pm}(t-z) .
\end{gathered}
$$

\section{B. Green function solution in four dimensions}

The Green function Eq. (30) is valid for sources that are spatially uniform in the $x$ and $y$ directions. In many situations, including laser pulses and the light from distant stars, this approximation is sufficient. However, in other cases the fully four-dimensional Green function may be relevant. As we show in this section, most of the 4D solution can be written in terms of the 2D Green function derived in Sec. II A. One of the new integrals cannot be so easily solved analytically, but with some effort it can be put in the form of a rapidly converging infinite series for easier numerical evaluation.

In the Lorenz gauge with neutral sources, $\nabla \cdot \mathbf{A}=0$, $A^{0}=0$, the Green function for $\mathbf{A}$ satisfies

$\left(\delta_{i j} \square-\dot{\theta} \epsilon_{\ell i k} \nabla_{\ell}\right) G_{k j}\left(x^{\mu}-y^{\mu}\right)=\delta_{i j} \delta^{(4)}\left(x^{\mu}-y^{\mu}\right)$,

where the cross product term in Eq. (5) forces the Green function $G_{k j}$ to have a nontrivial tensor structure. Its Fourier transform $\tilde{G}_{k j}$ satisfies

$$
\left[\left(-\omega^{2}+k^{2}\right) \delta_{i k}+2 i \mu k_{\ell} \epsilon_{\ell i k}\right] \tilde{G}_{k j}(\omega, \mathbf{k})=\delta_{i j},
$$

in terms of $\mu$ from Eq. (16), frequency $\omega$, and $k^{2}=k_{i} k^{i}$ for $i=1,2$, 3. Inverting the operator that acts on $\tilde{G}_{k j}$, the Green function can be written as

$$
\tilde{G}_{k j}=\tilde{A} \delta_{k j}+\tilde{B} k_{\ell} \epsilon_{\ell k j}+\tilde{C} k_{k} k_{j}
$$

where

$\tilde{A}=-\frac{\omega^{2}-k^{2}}{\beta}, \quad \tilde{B}=-\frac{2 i \mu}{\beta}, \quad \tilde{C}=\frac{4 \mu^{2}}{\left(\omega^{2}-k^{2}\right) \beta}$,

$\beta=\left(\omega^{2}-k^{2}\right)^{2}-4 k^{2} \mu^{2}$,

where the four roots of $\beta$ are

$$
\omega_{ \pm}^{2}=k^{2} \pm 2 k \mu .
$$


Since the current sources are transverse, the Green function can be simplified by the transverse projection

$$
\begin{aligned}
\tilde{G}_{i j}^{T} & \equiv\left(\delta_{i k}-\frac{k_{i} k_{k}}{k^{2}}\right) \tilde{G}_{k j}, \\
& =\tilde{A}\left(\delta_{i j}-\frac{k_{i} k_{j}}{k^{2}}\right)+\tilde{B} k_{\ell} \epsilon_{\ell i j}, \\
G_{i j}^{T} & =\delta_{i j} A+i \epsilon_{\ell i j} \nabla_{\ell} B+A_{i j},
\end{aligned}
$$

where $A, B$ and $A_{i j}$ are the Fourier transforms of $\tilde{A}, \tilde{B}$, and $-\tilde{A} k_{i} k_{j} / k^{2}$, respectively.

Both $A$ and $B$ can be written in terms of the scalar function $G_{0}(t, z)$ from the two-dimensional case, Eq. (30),

$$
\begin{aligned}
A & =\frac{1}{2 \pi r} \partial_{r}\left(\cos (r \mu) G_{0}\left(t, r ;-\mu^{2}\right)\right), \\
B & =\frac{\sin (r \mu)}{2 \pi r}\left(G_{0}\left(t, r ;-\mu^{2}\right)\right),
\end{aligned}
$$

where

$$
G_{0}\left(t, r ;-m^{2}\right) \equiv \frac{1}{2} \Theta(t) \Theta\left(t^{2}-r^{2}\right) I_{0}\left(\sqrt{m^{2} t^{2}-m^{2} r^{2}}\right) .
$$

Attempting the same technique for $A_{i j}$ leads to the incomplete expression

$$
A_{i j}=\frac{1}{4 \pi} \partial_{i} \partial_{j} \int_{-1}^{1} d q \cos (q r \mu) G_{0}\left(t, q r ;-\mu^{2}\right),
$$

an integral that does not have a simple expression in terms of Bessel functions or other hypergeometric functions. In Appendix B we show how the integral form of $A_{i j}$ can be replaced with an infinite series over a product of hypergeometric functions, with the result

$$
\begin{aligned}
A_{i j}= & \frac{1}{8 \pi} \partial_{i} \partial_{j}\left[\Theta(t) \Theta\left(t^{2}-r^{2}\right) \sum_{\ell=0}^{\infty} \frac{\left(-\frac{1}{4} \mu^{2} r^{2}\right)^{\ell}}{\ell !\left(\ell+\frac{1}{2}\right)}\left(\frac{2}{|\mu t|}\right)^{\ell}\right. \\
& \left.\times I_{\ell}(|\mu t|)_{1} F_{2}\left(\begin{array}{c}
\ell+\frac{1}{2} \\
\frac{1}{2}, \frac{\ell}{2}+\frac{1}{4}
\end{array} \mid-\frac{1}{4} \mu^{2} r^{2}\right)\right] .
\end{aligned}
$$

The series in $\ell$ converges rapidly for $\mu r \leq 2$. For large $\mu r \gg 1$ and $\mu t \gg 1$ it converges for $\ell>\ell_{\max }$, for an $\ell_{\max }(\mu t, \mu r)$ given in Appendix B.

\section{Application to monochromatic signals}

The Green function for the vector potential A [Eq. (30)] and its derivatives exhibit novel inside-the-light-cone components, which induce exponentially growing, semistatic residual fields in the wake of a signal. The on-thelight-cone contribution to the signal is modified as well, as a result of the perturbed phase velocity. Both of these effects provide signatures of the axion background in the path of an electromagnetic wave. In this section, we provide a few examples to show how simple monochromatic signals can be distorted on timescales $T$ that are shorter than the period of axion oscillation, $T \ll m_{a}^{-1}$.

\section{Phase velocity}

One distinctive feature of the modified electrodynamics, the helicity-dependent phase velocities, can be quantified directly from the equations of motion as in Refs. [29,30]. From the dispersion relations for right- and left-polarized light, Eq. (10), the phase velocities can be expanded in powers of $\mu=\frac{1}{2} \dot{\theta}(t)$,

$v_{\text {phase }}=\frac{\omega_{ \pm}}{k}=\sqrt{1 \pm 2 \frac{\mu}{k}}=1 \pm \frac{\mu}{k}-\frac{1}{2} \frac{\mu^{2}}{k^{2}}+\mathcal{O}\left(\mu^{3} / k^{3}\right)$.

This result can be also be recovered from the Green function in Eq. (30). If the signal is driven by a monochromatic source,

$$
j_{ \pm}(\tau)=e^{i \Omega \tau} f_{ \pm}(\tau)
$$

the solutions for $A_{ \pm}(t, z)$ are proportional to trigonometric factors of $\exp (i \Omega(t-z) \pm \mu z)$, reproducing the linear order term in Eq. (47).

Extremely sensitive measurements of the phase shift induced by gravitational waves are the bedrock for the remarkable recent detections of black hole and neutron star mergers. The sensitivity of advanced LIGO [47] to the gravitational strain $h=\Delta L / L$ exceeds $10^{-23} / \sqrt{\mathrm{Hz}}$ for gravitational waves with frequency $f \sim 10^{2} \mathrm{~Hz}$. An axion interferometer comparing the phases of left- and rightpolarized laser beams of angular frequency $\Omega$ observes a phase difference

$$
\Delta \phi \equiv \phi_{+}-\phi_{-}=2 \mu L=\dot{\theta}(t) L
$$

after the laser propagates a length $L$. Compared to the equivalent phase shift corresponding to a change in the path length $\Delta L, \Delta \phi=\Omega \Delta L$, the technology capable of detecting an $h_{\min } \sim 10^{-23}$ would also be able to set a limit on $\mu / \Omega$ of order

$$
\frac{2 \mu}{\Omega} \lesssim h_{\min }
$$

in the context of an axion interferometer. To use the $1064 \mathrm{~nm}$ laser of LIGO [48] as an example $(\Omega=1.77 \times$ $10^{15} \mathrm{rad} / \mathrm{s}$ ), an interferometer capable of similar precision has a potential sensitivity to any $\dot{\theta} \gtrsim 2 \times 10^{-8} \mathrm{~Hz}$, which 


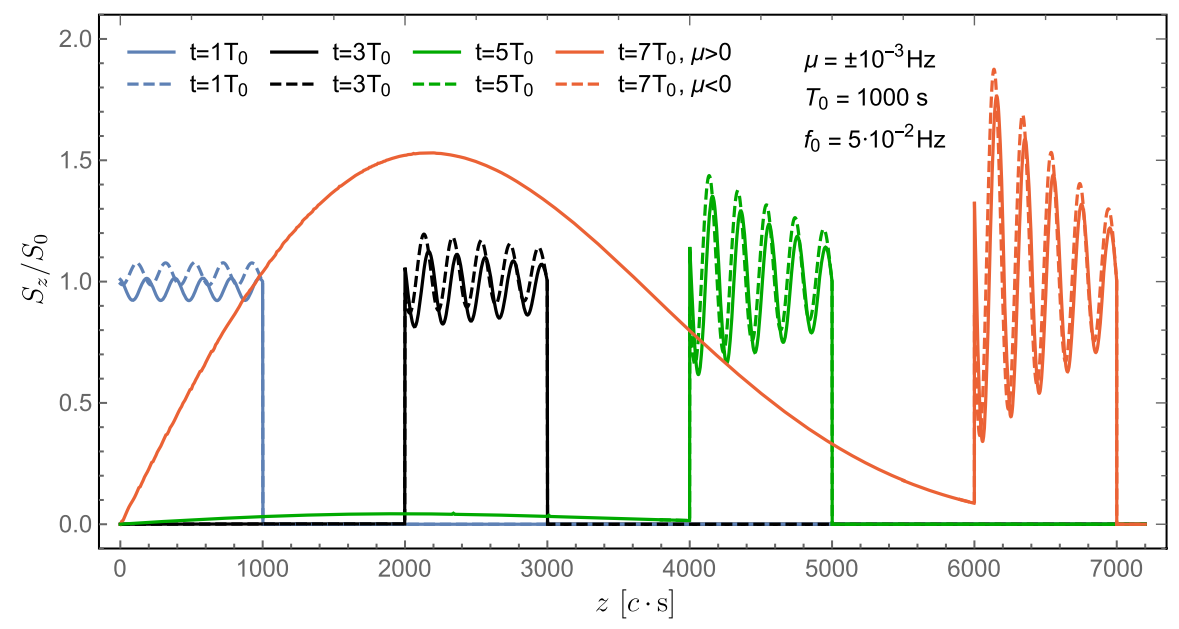

FIG. 2. The spatial profile the Poynting vector $S_{z}(z)$ of a propagating right-polarized square pulse is shown as a function of $z$, at several snapshots in time: $t=\left\{T_{0}, 3 T_{0}, 5 T_{0}, 7 T_{0}\right\}$ with $T_{0}=10^{3} \mathrm{~s}$, where the solid and dashed lines correspond, respectively, to $\mu= \pm 10^{-3} \mathrm{~Hz}$. For $0 \leq t-z \leq T_{0}$ ("on the light cone"), the difference in sign affects the phase velocities and spectrum of the pulses, but for $t-z>T_{0}$ ("inside the light cone") the sign of $\mu$ is irrelevant for $S_{z}(t, z)$.

overlaps with the parameter space indicated by Eq. (7) for $10^{-11} \mathrm{GeV}^{-1} \lesssim g_{\text {ary }} \lesssim 10^{-10} \mathrm{GeV}^{-1}$.

To achieve the sensitivity indicated by Eq. (50), the approximation that $\dot{\theta}(t)=2 \mu$ is constant needs to hold only for as long as it takes the photon beam to traverse the interferometer. As long as $m_{a} L \ll 1$ and $m_{a} T \ll 1$ for the characteristic length $L$ and time of flight $T$ for the measurement, it is not necessary to insist that $m_{a} \ll \dot{\theta}$. A detector with $c T \sim n L \sim \mathcal{O}\left(10^{3} \mathrm{~km}\right.$ ) (where $n$ is the effective number of reflections of the light within the chamber) would thus be sensitive to $m_{a} \lesssim 10^{-12} \mathrm{eV}$, while more massive axions would be more easily detected with shorter interferometers. In the case of LIGO, the beam cavity storage time $T$ is long compared to the length of each arm, with $T \sim n L / c$ for some $n \approx 70$ [48].

\section{After-pulse residual fields}

A substantively new effect appears when we consider the Green function to all orders in $\dot{\theta}$, for signals of finite duration $T$. In the $\dot{\theta}=0$ vacuum of standard electrodynamics, such a signal propagates away from the source at speed $c$, maintaining the same duration $T$. In the $\dot{\theta} \neq 0$ background, this is no longer true. Rather than returning to zero after the signal passes, the $\mathbf{E}$ and $\mathbf{B}$ fields retain a residual nonzero value that grows with time. Interpreting $-\frac{1}{4} \dot{\theta}^{2}$ as a tachyonic mass term for the photon, the growth of the $\mathbf{E}$ and $\mathbf{B}$ fields is a consequence of the tachyonic instability - a transfer of energy from the axion background into long-wavelength photons triggered by the original signal. This growth takes place inside the light cone, rather than strictly on it.

Again specializing to a monochromatic signal $j_{ \pm}(\tau)=$ $2 e^{i \Omega \tau} f_{ \pm}(\tau)$ for simplicity, and taking the source at $z=0$ to satisfy $j_{ \pm}(\tau)=0$ for $\tau<0$ and $\tau>T$, the "residual" field $A_{ \pm}^{(r)}$ at $z>0$ refers to the nonzero field value after $t-z>T$. We have

$$
\begin{aligned}
& \partial_{t} A_{ \pm}^{(r)}(t-z>T) \\
& \quad=\mu \int_{0}^{T} d \tau e^{i \Omega \tau \pm i \mu z} f_{ \pm}(\tau) \frac{(\mu t-\mu \tau) I_{1}\left(\sqrt{\lambda^{\prime}}\right)}{\sqrt{\lambda^{\prime}}},
\end{aligned}
$$

$$
\begin{aligned}
& \partial_{z} A_{ \pm}^{(r)}(t-z>T) \\
& \quad=\mu \int_{0}^{T} d \tau e^{i \Omega \tau \pm i \mu z} f_{ \pm}(\tau)\left( \pm i I_{0}\left(\sqrt{\lambda^{\prime}}\right)-\frac{\mu z I_{1}\left(\sqrt{\lambda^{\prime}}\right)}{\sqrt{\lambda^{\prime}}}\right)
\end{aligned}
$$

where $\lambda^{\prime}=\mu^{2}(t-\tau)^{2}-\mu^{2} z^{2}$. In the high frequency limit, the rapid oscillations of $e^{i \Omega \tau}$ tend to cancel out the contributions from both integrals, so that the strengths of the residual $\mathbf{E}$ and $\mathbf{B}$ fields are proportional to $\mu / \Omega$. However, especially for $\mu T \sim \mathcal{O}(1)$, the residual fields can become substantial: for $\lambda^{\prime} \gtrsim \mathcal{O}(1)$, the exponential growth of the Bessel functions becomes apparent, and eventually compensates for the $\mu / \Omega$ suppression.

To demonstrate the distortion to a signal as it propagates through space, Fig. 2 shows the Poynting vector $\mathbf{S}=\mathbf{E} \times$ $\mathbf{B}$ as a function of $z$ at four snapshots in time. At $t=T_{0}$, when the signal is newly produced, it exhibits relatively mild modifications to the original $S_{z}(t, z)=S_{0}$ square wave, in this example with $T_{0}=\mu^{-1}$. By the $t=5 T_{0}$ snapshot, not only has the signal become notably distorted, but it also has developed nonzero values inside the light cone, of magnitude $S_{z}\left(t-z>T_{0}\right) \sim 10^{-1} S_{0}$. At $t=7 T_{0}$ this part of the field exceeds $S_{z}\left(t-z>T_{0}\right) \gtrsim S_{0}$, and continues to grow exponentially for $t>7 T_{0}$. 

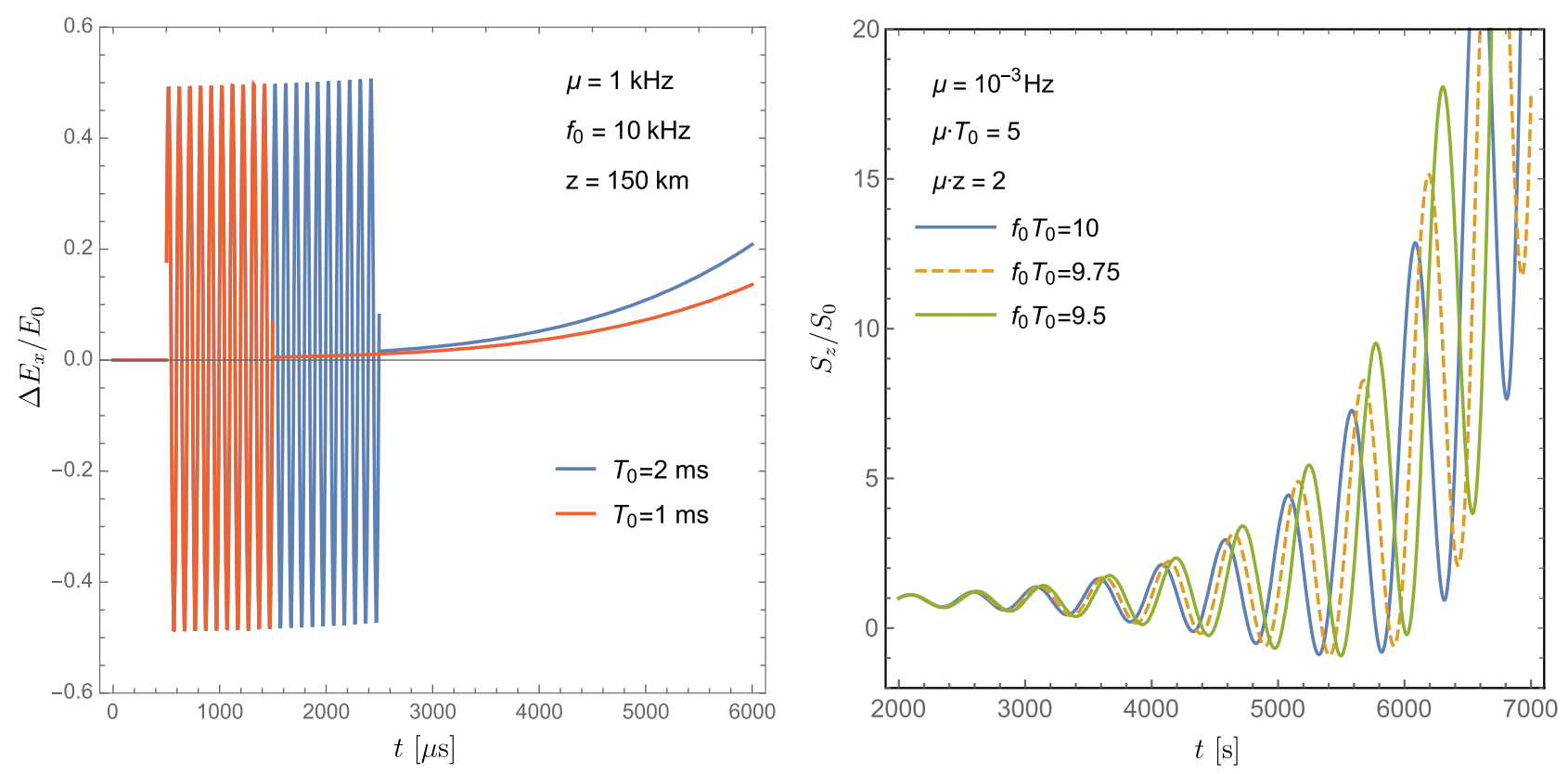

FIG. 3. Left: the $E_{x}$ field is shown as a function of time at fixed $z=150 \mathrm{~km}$ for square pulses of frequency $f_{0}=\Omega / 2 \pi=10 \mathrm{kHz}$, and durations $1000 \mu \mathrm{s}$ (orange) and $2000 \mu \mathrm{s}$ (blue). Oscillations begin as the front of the pulse passes through the fixed value of $z$, followed at later times by monotonic growth. For illustration we use an inflated value of $\mu=1 \mathrm{kHz}$. Not pictured, the $\mathbf{B}$ and $E_{y}$ fields have a nearly identical profile. Right: the value of the Poynting vector $S_{z}$ is shown as a function of time for three square pulses of duration $T_{0}=5 \times 10^{3} \mathrm{~s}$ and similar frequencies $f_{0} \sim 10 / T_{0}$, measured at a distance $z=2 / \mu$ from the source, for $\mu=10^{-3} \mathrm{~Hz}$. In the $\mu=0$ case the Poynting vector would be constant, with $S_{z}^{m=0}\left(0 \leq t-z \leq T_{0}\right)=S_{0}$.

In Fig. 3, we show $E_{x}(t)$ for two square pulses with different durations $T$, to demonstrate the relationship between the pulse length and the strength of the residual fields. In this example we inflate the typical value of $\mu$ to $\mu=1 \mathrm{kHz}$ so that the strength of the residual pulse approaches the amplitude of the original signal for $t \sim \mathcal{O}$ (few) $\times T$ in an example with $10 \mathrm{kHz}$ radiation. However, the same plots can be reused for any value of $\mu$ by rescaling $\Omega, t$ and $z$ so as to keep $\mu \Omega, \mu t$ and $\mu z$ constant, as in the right-hand panel of Fig. 3. For another example, using $\mu=10^{-8} \mathrm{~Hz}$ instead of $10^{3} \mathrm{~Hz}$, the plot in Fig. 3 would show $t$ in units of $10^{5} \mathrm{~s}$ rather than $\mu \mathrm{s}$, and $z=1.5 \times 10^{13} \mathrm{~km}=0.49 \mathrm{pc}$.

\section{Exponential growth}

At sufficiently late times, $\mu t \gtrsim 1$, the growth in the $\mathbf{E}$ and B fields highlighted in Fig. 3 becomes exponential, driven by the Bessel functions $I_{0}(\sqrt{\lambda})$ and $\lambda^{-1 / 2} I_{1}(\sqrt{\lambda})$ in the Green functions Eqs. (51)-(52). This is true not only of the semistatic residual fields in the wake of the signal, but also for the signal itself, if its duration $T$ is long compared to $\mu^{-1}$. For the phenomenologically relevant values of $\mu$ given by Eq. (7), probing the strongly exponential behavior requires extremely long coherence times $T_{0}>\mu^{-1} \gtrsim$ $10^{8} \mathrm{~s}$ for both the axion field and the radiation source, unless the axion density is significantly enhanced beyond the expected $\rho_{a} \sim 0.4 \mathrm{GeV} / \mathrm{cm}^{3}$. Nevertheless, it is a useful exercise to explore the behavior of the fields in this extreme limit.

Even in the late-time limit $\mu(t-z)>1$, there is a clear distinction between the received "signal," when $0 \leq t-z \leq T$, and the "residual" fields, $t-z>T$. In contrast to the relatively mild modifications to the signal in Fig. 3, the signal eventually becomes significantly amplified and distorted for large $\mu t \gg 1$ and $\mu z \gg 1$. The semistatic residual fields that appear in the wake of the signal continue to grow exponentially, until the point where the energy density in the electromagnetic fields becomes comparable to $\rho_{a}$, and the backreaction on the axion field can no longer be neglected.

Figure 3 (right panel) shows the modified signal for an example with $\mu=10^{-3} \mathrm{~Hz}$, where the pulse duration $\left(T=5 \times 10^{3} \mathrm{~s}\right)$ and propagation distance $\left(z=6 \times 10^{8} \mathrm{~km}\right)$ are both larger than $\mu^{-1}$. In the $\mu \rightarrow 0$ limit of standard electrodynamics, the power density $\left|S_{z}\right|$ of this circularly polarized square wave would remain constant, $S_{z}^{m=0}(0 \leq$ $t-z \leq T)=S_{0}$, returning to zero for $t-z>T$. Instead, the received power in the $\mu \neq 0$ case varies as a function of time, oscillating with ever-larger fluctuations and increasing exponentially. Once $\mu(t-z) \gtrsim 1$, the fluctuations in the power $\Delta S_{z}$ become larger than the magnitude of the power at the source, $S_{0}$, and the exponential growth soon ensures that $S_{z}(t) \gg S_{0}$ for all $t-z \gg \mu^{-1}$.

After the signal has passed by, the residual fields are well described by Eqs. (51)-(52), which in the $\lambda^{\prime} \gg 1$ limit 

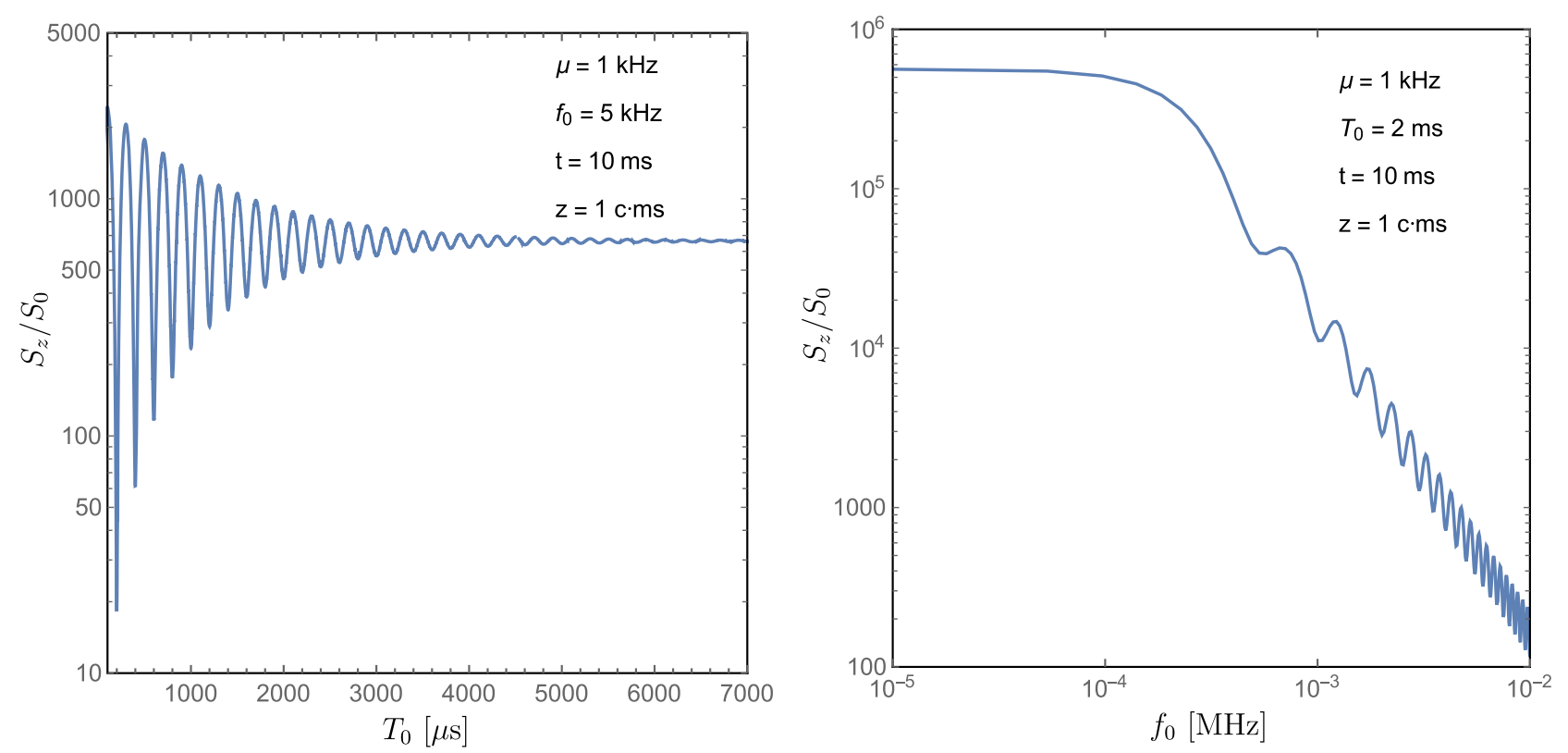

FIG. 4. Left: the fractional change in the radiation power $S_{z}$ at $z=300 \mathrm{~km}$, for $\mu=1 \mathrm{kHz}$, as a function of pulse duration $T_{0}$, measured at $t=T_{0}+10 \mathrm{~ms}$. The approach to a constant value as the pulse duration increases reflects the fact that it is the first part of the signal which has induced the largest contribution to the growing instability. Right: $\Delta S_{z} / S_{0}$ as a function of radiation frequency $f=\Omega / 2 \pi$, for the same values of $\mu$ and $z$, for fixed $t=10 \mathrm{~ms}$ and $T_{0}=2 \mathrm{~ms}$. The falling value of $\Delta S_{z}$ mimics the $\Delta S_{z} \propto \Omega^{-2}$ scaling indicated in Sec. II C 3. The oscillations in the received power for $t-z>T_{0}$ correspond to integer values of $\pi \Omega T_{0}$. As shown in the left panel, small variations in $\Omega T_{0}$ have a substantial impact on the power when $\mu T_{0} \lesssim \mathcal{O}(\mathrm{few})$, but for larger values of $T_{0}$ the contribution to $S_{z}(t, z)$ from the latter part of the signal is dwarfed by the exponential growth seeded by the first part.

closely resemble the asymptotic expansion described in Eq. (B11) in Appendix B. The dominant term in this expansion is the exponential

$$
\lim _{\lambda \rightarrow \infty} I_{j}(\sqrt{\lambda}) \approx \frac{e^{\sqrt{\lambda}}}{\sqrt{2 \pi \sqrt{\lambda}}} .
$$

To show the dependence of the strength of the residual fields on the pulse length $\Omega T_{0}$, the left panel of Fig. 4 shows $S_{z}(t, z)$ at $t=10 \mathrm{~ms}, z=1 \mathrm{~ms}$, as a function of pulse duration $T_{0}$, with fixed frequency $f_{0}=\Omega / 2 \pi=$ $5 \mathrm{kHz}$. Once $T_{0} \gtrsim 4 \mu^{-1}$, the strength of the field at $(t, z)$ approaches a constant value. Evidently, $S_{z}(t-z>T)$ is driven primarily by the first part of the signal, which has induced the longest-lived instabilities: the subsequent exponential growth of the initial instability makes this part of the signal the most consequential.

By considering the high-frequency limit of Eqs. (51)(52), where $f_{ \pm}(\tau)$ and $I_{j}\left(\sqrt{\lambda^{\prime}}\right)$ both vary slowly compared to $e^{i \Omega \tau}$, it is easy to see that the inside-the-light-cone electromagnetic fields scale as $|\mathbf{E}| \propto \Omega^{-1}$ and $|\mathbf{B}| \propto \Omega^{-1}$, so that the radiation density is proportional to $S_{z} \propto \Omega^{-2}$. In the right panel of Fig. 4, the power $S_{z}(t, z)$ is shown for the same values of $t$ and $z$, this time as a function of the frequency of the square pulse $\left(f_{0}=\Omega / 2 \pi\right)$, with fixed pulse duration $T_{0}=2 \times 10^{-3} \mathrm{~s}$. At low frequencies, $f_{0} \ll T_{0}^{-1}$, the power approaches a constant. In this limit the phases of the integrands in Eqs. (51)-(52) are essentially independent of $\Omega, e^{i \Omega \tau \pm i \mu z} \approx e^{ \pm i \mu z}$. In the opposite limit, $f_{0} \gg T_{0}^{-1}$, the $S_{z} \propto \mu^{2} / \Omega^{2}$ scaling suggested by Eqs. (51)-(52) becomes manifest. Together, the $T_{0} \gg$ $\mu^{-1}$ and $f_{0} \gg \mu$ limits indicate that the power scales roughly as $S_{z}(t, z) \sim e^{\sqrt{\lambda}}(\mu / \Omega)^{2}$, for $\lambda=\mu^{2} t^{2}-\mu^{2} z^{2}$ with $t-z \gg T_{0}$. In many applications involving radio, visible or X-ray radiation, the hierarchy between $\mu$ and $\Omega$ imposes an extreme penalty on the magnitude of the surplus power, so that only after multiple $e$-foldings would it be possible to detect the signal.

Our derivation of the Green function Eq. (30) assumes that $\ddot{\theta}$ vanishes, so that $\mu(t)$ can be treated as constant. For a standard axion oscillating in a quadratic potential, $\mu(t)$ varies according to

$$
\mu(t)=\mu_{0} \cos \left(m_{a} t\right), \quad \mu_{0}=\frac{1}{2} g_{a \gamma \gamma} \sqrt{\rho_{a}} .
$$

At times $T$ comparable to the period of axion oscillation, $T \sim m_{a}^{-1}$, the steady-state approximation $\mu(t) \simeq \mu$ is no longer valid, and it is necessary to use the methods described in Sec. III. In principle, because $\mu_{0}$ is set directly by $\rho_{a}$ and $g_{a \gamma \gamma}$, it is independent of $m_{a}$, and the exponential growth in the late-time limit $\mu T \gg 1$ can be explored without violating the steady-state condition $m_{a} T \ll 1$. Achieving $\mu_{0} \gg m_{a}$ does, however, require a nonstandard 
ALP model with an extended field range. Equation (54) implies

$$
\mu(t)=\mu_{0} \cos \left(m_{a} t\right) \rightarrow \theta(t)=\theta_{0} \sin \left(m_{a} t\right), \quad \theta_{0}=2 \frac{\mu_{0}}{m_{a}},
$$

so $\mu_{0} \gg m_{a}$ necessarily implies that $\theta_{0} \gg 2$.

In practice, this region of parameter space with $\mu_{0}>m_{a}$ is mostly ruled out by experiment. Based on the constraints for $g_{\text {ary }}[10-15]$ and $m_{a}$ [38-41], for dark matter densities of $\rho_{a} \approx 0.4 \mathrm{GeV} / \mathrm{cm}^{3}$, Eq. (7) indicates that $\dot{\theta} \ll m_{a}$ for all allowed $m_{a}$ and $g_{a \gamma \gamma}$. To realize the late-time exponential growth within the steady-state axion background, three conditions must be satisfied: $m_{a}$ must be near the low-mass "fuzzy dark matter" extreme, $m_{a} \sim 10^{-21} \mathrm{eV}$; the coupling $g_{a \gamma \gamma}$ must be relatively strong, $g_{a \gamma \gamma} \lesssim 10^{-11} \mathrm{eV}$, and the value of $\sqrt{\rho_{a}}$ in the path of the photon must be enhanced by a few orders of magnitude, for example by concentrating some fraction of the axions into dense clumps or "axion stars." If these conditions are not satisfied, then $m_{a} T \ll 1$ implies $\mu_{0} T \ll 1$, and the Green function is well approximated by its series expansion in $\lambda$.

\section{OSCILLATING AXION BACKGROUND}

In an oscillating axion background,

$$
\dot{\theta}(t)=\dot{\theta}_{0} \cos \left(m_{a} t\right), \quad \dot{\theta}_{0}=g_{a \gamma \gamma} \sqrt{\rho_{a}},
$$

the Green function for the vector potential $A_{ \pm}$in $(1+1)$ dimensional spacetime satisfies

$$
\begin{aligned}
& \left(\partial_{t}^{2}-\partial_{z}^{2} \pm i \dot{\theta}_{0} \cos \left(m_{a} t\right) \partial_{z}\right) g_{ \pm}\left(t-t_{0}, z-z_{0}\right) \\
& \quad=\delta\left(z-z_{0}\right) \delta\left(t-t_{0}\right)
\end{aligned}
$$

As in Sec. II A we restrict our analysis to propagating plane waves. Unlike the $\dot{\theta} \approx$ const limit of Eq. (19), Eq. (57) cannot be inverted to find an algebraic expression for the Fourier transform of $g_{ \pm}$. Instead,

$$
\begin{aligned}
& \left(k^{2}-\omega^{2}\right) \tilde{g}_{ \pm}(\omega, k) \\
& \mp \frac{k \dot{\theta}_{0}}{2}\left(e^{i t_{0} m_{a}} \tilde{g}_{ \pm}\left(\omega+m_{a}, k\right)+e^{-i t_{0} m_{a}} \tilde{g}_{ \pm}\left(\omega-m_{a}, k\right)\right)=1
\end{aligned}
$$

couples $\tilde{g}_{ \pm}(\omega)$ to $\tilde{g}_{ \pm}\left(\omega \pm m_{a}\right)$. This complication stems from the fact that the solutions of the homogeneous equations of motion are Mathieu functions.

In both of the limits $\dot{\theta}_{0} T \gg 1$ and $\dot{\theta}_{0} T \ll 1$, the leading forms of the Green functions can be extracted from Eq. (58) without invoking Mathieu functions or their Fourier transforms. Section III A focuses on the former limit, in which frequencies $\omega=\frac{1}{2} m_{a} \pm \mathcal{O}\left(\dot{\theta}_{0}\right)$ are resonantly enhanced, inducing exponential growth for these unstable frequencies. In the alternate limit $\dot{\theta}_{0} T \ll 1$ where the exponential growth is not realized, and for $\dot{\theta}_{0} T \gtrsim 1$ for signals that do not include the resonantly enhanced frequencies, Sec. III B provides a continued fraction expression for the Green function that converges quickly for small $\dot{\theta}_{0} \ll m_{a}$.

\section{A. Resonantly enhanced propagation}

For small $\dot{\theta}_{0} \ll m_{a}$, Eq. (58) suggests that the solution for $\tilde{g}_{ \pm}(\omega)$ could be found as an expansion in $k \dot{\theta}_{0} /\left(k^{2}-\omega^{2}\right)$. However, for $\omega \approx k \pm \mathcal{O}\left(\dot{\theta}_{0}\right)$ the simple perturbative expansion is disrupted, especially near frequencies $\omega= \pm \frac{1}{2} m_{a}$ where $\omega^{2}=\left(\omega \pm m_{a}\right)^{2}$.

At late times, when $\dot{\theta}_{0} T \gtrsim 1$, the exponential growth of the unstable modes with $\omega= \pm m_{a} / 2+\mathcal{O}\left(\dot{\theta}_{0}\right)$ dominates the propagation of a signal. In this late-time limit the Green function can be approximated by integrating over the resonantly enhanced modes, ${ }^{2}$

$g_{ \pm}\left(t \mid t_{0}, z\right) \approx \sum_{k, \omega \approx \pm m_{a} / 2} \int \frac{d k d \omega e^{i k z-i \omega\left(t-t_{0}\right)}}{(2 \pi)^{2}} G\left(\omega_{ \pm}, k_{ \pm}\right)$,

where we expand $\omega$ and $k$ about $\pm m_{a} / 2$, defining

$\epsilon \equiv \pm \frac{\dot{\theta}_{0}}{4 m_{a}}, \quad k_{ \pm} \equiv \pm \frac{m_{a}}{2}+\alpha \epsilon, \quad \omega_{ \pm} \equiv \pm \frac{m_{a}}{2}+\beta \epsilon$

The factor of $\pm \dot{\theta}_{0}$ in Eq. (57) corresponding to right- and left-polarized light is absorbed into the definition of the parameter $\epsilon$. Even in the corner of ALP parameter space with small masses and relatively large couplings, Eq. (7) indicates that $\epsilon \lesssim 10^{-1}$ remains perturbatively small. For ALPs more closely resembling a QCD axion with $m_{a}>10^{-12} \mathrm{eV}, g_{\text {ary }}<10^{-11} \mathrm{GeV}^{-1}$, and fixed $\rho_{a} \approx$ $0.4 \mathrm{GeV} / \mathrm{cm}^{3}$, the value of $\epsilon$ drops to $\epsilon<10^{-11}$.

The function $G\left(\omega_{ \pm}, k_{ \pm}\right)$introduced in Eq. (59) is defined to include only the $\mathcal{O}\left(\epsilon^{-1}\right)$ part of $\tilde{g}_{ \pm}$in the neighborhood of the resonant frequencies, where $\alpha$ and $\beta$ are $\mathcal{O}\left(m_{a}\right)$. By dropping the $\mathcal{O}\left(\epsilon^{0}\right)$ portion of the Green function, Eq. (58) can be disentangled to solve for $G\left(\omega_{ \pm}, k_{ \pm}\right)$,

$$
\begin{aligned}
& G\left(\omega_{ \pm}, k_{+}\right)=\frac{1}{\epsilon m_{a}}\left(\frac{\alpha \pm \beta+m_{a} e^{\mp i t_{0} m_{a}}}{\alpha^{2}-\beta^{2}-m_{a}^{2}}\right), \\
& G\left(\omega_{ \pm}, k_{-}\right)=-\frac{1}{\epsilon m_{a}}\left(\frac{\alpha \mp \beta+m_{a} e^{\mp i t_{0} m_{a}}}{\alpha^{2}-\beta^{2}-m_{a}^{2}}\right) .
\end{aligned}
$$

\footnotetext{
${ }^{2}$ Here we employ a multiscale technique used in Ref. [49] in an asymptotic analysis of lattice Green functions.
} 
In terms of $\alpha$ and $\beta$, Eq. (59) reduces to

$$
\begin{aligned}
g_{ \pm}\left(t \mid t_{0}, z\right) \approx & \int \frac{4 \epsilon^{2} d \alpha d \beta e^{i \epsilon \alpha z-i \epsilon \beta\left(t-t_{0}\right)}}{(2 \pi)^{2} \epsilon m_{a}\left(\alpha^{2}-\beta^{2}-m_{a}^{2}\right)} \times\left(i \alpha \cos \frac{m_{a}\left(t-t_{0}\right)}{2} \sin \frac{m_{a} z}{2}-i \beta \sin \frac{m_{a}\left(t-t_{0}\right)}{2} \cos \frac{m_{a} z}{2}\right. \\
& \left.+i m_{a} \cos \frac{m_{a}\left(t+t_{0}\right)}{2} \sin \frac{m_{a} z}{2}\right)+\mathcal{O}\left(\epsilon^{0}\right) .
\end{aligned}
$$

As always, the retarded Green function is defined to satisfy $g\left(t<t_{0}\right)=0$, so the contour in $\omega(\beta)$ passes above both poles at $\beta= \pm \sqrt{\alpha^{2}-m_{a}^{2}}$, even when $\alpha^{2}<m_{a}^{2}$.

Fortuitously, this integral is nearly identical to the one encountered in Sec. II. Following the example of Eqs. (22)-(23), we introduce the coordinate transformations

$$
\begin{gathered}
\alpha \equiv m_{a} \cosh \varphi, \quad \beta_{0} \equiv+\sqrt{\alpha^{2}-m_{a}^{2}}=+i \sqrt{m_{a}^{2}-\alpha^{2}} \equiv m_{a} \sinh \varphi, \\
m_{a} \epsilon z=\sqrt{\lambda} \sinh \eta, \quad m_{a} \epsilon\left(t-t_{0}\right)=\sqrt{\lambda} \cosh \eta, \quad \lambda=m_{a}^{2} \epsilon^{2}\left(\left(t-t_{0}\right)^{2}-z^{2}\right) .
\end{gathered}
$$

As in Fig. 1, $\varphi$ is such that $\cosh \varphi$ runs smoothly from $-\infty$ to $+\infty$ with $\operatorname{Im} \cosh (\varphi)=0$. Extending the limits of integration in Eq. (62) to $-\infty<\beta<\infty$ and $-\infty<\alpha<\infty$, we find

$$
\begin{aligned}
g\left(t \mid t_{0}, z\right) \approx & \frac{\Theta\left(t-t_{0}\right) \Theta\left(\left(t-t_{0}\right)^{2}-z^{2}\right)}{2}\left(1+4 i \epsilon \cos \frac{m_{a}\left(t+t_{0}\right)}{2} \sin \frac{m_{a} z}{2} I_{0}(\sqrt{\lambda})\right. \\
& \left.+4 \epsilon\left[m_{a} \epsilon\left(t-t_{0}\right) \sin \frac{m_{a}\left(t-t_{0}\right)}{2} \cos \frac{m_{a} z}{2}-m_{a} \epsilon z \cos \frac{m_{a}\left(t-t_{0}\right)}{2} \sin \frac{m_{a} z}{2}\right] \frac{I_{1}(\sqrt{\lambda})}{\sqrt{\lambda}}\right),
\end{aligned}
$$

which is again causal and exhibits propagation inside the light cone. The $\epsilon^{0}$ term in the expansion is calculated separately, by considering the Maxwell theory $\epsilon \rightarrow 0$ limit. In the late-time limit, when $T=\left(t-t_{0}\right)$ satisfies

$$
2 \epsilon m_{a} T+\log \epsilon \gg \log m_{a} T
$$

the $\epsilon I_{0}(\sqrt{\lambda})$ and $\epsilon I_{1}(\sqrt{\lambda})$ contributions become larger than $\mathcal{O}(1)$, and the resonantly enhanced modes dominate the Green function.

Compared to the $m_{a} \rightarrow 0$ limit from Sec. II, the approximate retarded Green function in the resonantly enhanced regime of $m_{a} \gg \dot{\theta}$ is remarkably similar. The exponential growth scale $\mu(t)$ has been replaced by

$$
m_{a} \epsilon=\frac{\mu_{0}}{2}=\frac{\dot{\theta}_{0}}{4},
$$

which is in line with what we naively expect from Sec. II. For example, if we used the steady-state result to approximate the late-time exponential growth by replacing $|\mu(t)|$ with its average value, $\langle|\mu(t)|\rangle=\frac{1}{\pi} \dot{\theta}_{0}$, the resulting estimate for the growth factor is off by only $27 \%$.

Recall from Eq. (7) that for fixed axion density $\rho_{a}=0.4 \mathrm{GeV} / \mathrm{cm}^{3}$, even in the corner of parameter space

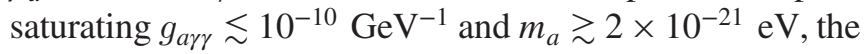
value of $\epsilon$ is still perturbatively small, $|\epsilon| \lesssim 2 \times 10^{-3}$. For this roughly maximal value of $\epsilon$, Eq. (66) is satisfied by $m_{a} T \gtrsim 3600$. Elsewhere in the $\left(m_{a}, g_{a \gamma \gamma}\right)$ parameter space, $\epsilon$ can assume significantly smaller values, requiring larger $m_{a} T \gg 10^{3}$ to satisfy Eq. (66).

Our treatment of the axion background as a coherently oscillating field requires the field to remain coherent throughout the signal propagation, or $T<T_{c}$ with $T_{c} \sim$ $\left(m_{a} v^{2}\right)^{-1}$, where $v \sim 10^{-3}$ is the virial velocity of the axions. In the case of photons traveling freely through space, as opposed to reflecting within some cavity, the propagation distance $L$ must also be smaller than some $L_{c} \sim\left(m_{a} v\right)^{-1}$. For $\epsilon \lesssim 10^{-5}$, the onset of exponential growth indicated by Eq. (66) requires $m_{a} T>10^{6} \sim 1 / v^{2}$, meaning that decoherence effects become important on the timescales associated with the exponential growth, and must be accounted for. This result is consistent with Ref. [45], which found that for axion models with $10^{-8} \mathrm{eV}<m_{a}$, decoherence completely obscures the exponential growth. Additionally, for these models the width is narrow enough that gravitational redshift by the dark matter halo is sufficient to detune the resonance, even if the velocity for the axion cloud is taken to be $v \ll 10^{-3}$ in order to satisfy $m_{a} T>1 / v^{2}$, leading the authors of Ref. [45] to conclude that for $m_{a}>$ $10^{-8} \mathrm{eV}$ in the observable range the parametric resonance at $\omega=\frac{1}{2} m_{a}$ never develops into exponential growth.

Nevertheless, there is a window where $\epsilon \gtrsim 10^{-5}$ occupying a couple decades of the $\left(m_{a}, g_{a \gamma \gamma}\right)$ parameter space in which the resonance can develop. It applies to extremely 
low-frequency radiation of $\omega \gtrsim 10^{-21} \mathrm{eV}$, or equivalently $f \gtrsim 10^{-7} \mathrm{~Hz} \sim 10 \mathrm{yr}^{-1}$, which does not propagate through the interstellar medium.

\section{B. Propagation without resonance}

In the opposite limit to Eq. (66), where $\epsilon m_{a} T \sim \dot{\theta} T \ll 1$ and the resonance is not given time to grow, an alternate approach provides a perturbative expansion of the Green function in powers of $\epsilon$. This approach is also valid in the $\epsilon m_{a} T>1$ limit for signals that do not include support within the instability band $\omega=m_{a} / 2 \pm \mathcal{O}(\dot{\theta})$, and generically for any signal where the resonant component from Eq. (65) can be approximated by its series expansion.

For values of $\omega \neq m_{a} / 2$, Eq. (58) can be rearranged into a continued fraction solution for $\tilde{g}_{ \pm}(\omega)$, as a generalization of the relation

$$
\tilde{g}_{ \pm}(\omega, k)=\frac{1}{k^{2}-\omega^{2}}+\frac{2 k m_{a} \epsilon}{k^{2}-\omega^{2}}\left(e^{i t_{0} m_{a}} \tilde{g}_{ \pm}\left(\omega+m_{a}, k\right)+e^{-i t_{0} m_{a}} \tilde{g}_{ \pm}\left(\omega-m_{a}, k\right)\right),
$$

in terms of $\epsilon$ from Eq. (60). By iterating the replacement of $\tilde{g}_{ \pm}\left(\omega \pm m_{a}\right)$ with $\tilde{g}_{ \pm}(\omega)$ and $\tilde{g}_{ \pm}\left(\omega \pm 2 m_{a}\right)$, we derive an approximation of the form $\tilde{g}_{ \pm}(\omega, k) \approx a_{\ell}(\omega, k)$ as follows:

$$
\begin{aligned}
\tilde{g}_{ \pm}(\omega) & =a_{0}(\omega)+b_{0}(\omega) \tilde{g}_{ \pm}\left(\omega+m_{a}\right)+b_{0}(\omega) \tilde{g}_{ \pm}\left(\omega-m_{a}\right), \\
\tilde{g}_{ \pm}(\omega) & =a_{1}(\omega)+b_{1}(\omega) \tilde{g}_{ \pm}\left(\omega+2 m_{a}\right)+b_{-1}(\omega) \tilde{g}_{ \pm}\left(\omega-2 m_{a}\right), \\
& \vdots \\
\tilde{g}_{ \pm}(\omega) & =a_{\ell}(\omega)+b_{\ell}(\omega) \tilde{g}_{ \pm}\left(\omega+2^{\ell} m_{a}\right)+b_{-\ell}(\omega) \tilde{g}_{ \pm}\left(\omega-2^{\ell} m_{a}\right),
\end{aligned}
$$

where the $k$ dependence of each function $\tilde{g}_{ \pm}\left(\omega \pm 2^{\ell} m_{a}, k\right)$ has been left implicit, and where

$$
\begin{aligned}
a_{0}(\omega) & =\frac{1}{k^{2}-\omega^{2}}, \quad a_{1}(\omega)=\frac{a_{0}(\omega)+b_{+0}(\omega) a_{0}\left(\omega+m_{a}\right)+b_{-0}(\omega) a_{0}\left(\omega-m_{a}\right)}{1-b_{+0}(\omega) b_{-0}\left(\omega+m_{a}\right)+b_{-0}(\omega) b_{+0}\left(\omega-m_{a}\right)}, \\
b_{ \pm 0}(\omega) & =\frac{2 \epsilon k m_{a} e^{ \pm i m_{a} t_{0}}}{k^{2}-\omega^{2}}, \quad b_{ \pm 1}(\omega)=\frac{b_{ \pm 0}(\omega) b_{ \pm 0}\left(\omega \pm m_{a}\right)}{1-b_{+0}(\omega) b_{-0}\left(\omega+m_{a}\right)+b_{-0}(\omega) b_{+0}\left(\omega-m_{a}\right)} .
\end{aligned}
$$

The recursion is provided by

$$
\begin{aligned}
a_{\ell+1}(\omega) & =\frac{a_{\ell}(\omega)+b_{+\ell}(\omega) a_{\ell}\left(\omega+2^{\ell} m_{a}\right)+b_{-\ell}(\omega) a_{\ell}\left(\omega-2^{\ell} m_{a}\right)}{1-b_{+\ell}(\omega) b_{-\ell}\left(\omega+2^{\ell} m_{a}\right)-b_{-\ell}(\omega) b_{+\ell}\left(\omega-2^{\ell} m_{a}\right)}, \\
b_{ \pm(\ell+1)}(\omega) & =\frac{b_{ \pm \ell}(\omega) b_{ \pm \ell}\left(\omega \pm 2^{\ell} m_{a}\right)}{1-b_{+\ell}(\omega) b_{-\ell}\left(\omega+2^{\ell} m_{a}\right)-b_{-\ell}(\omega) b_{+\ell}\left(\omega-2^{\ell} m_{a}\right)} .
\end{aligned}
$$

The convergence of the continued fraction expression effectively depends on a small- $b$ expansion, and for generic values of $\omega \sim k \gg O\left(\epsilon m_{a}\right)$ counting powers of $\epsilon$ is relatively easy: all of the $a_{i}$ are $\mathcal{O}\left(\epsilon^{0}\right)$, while $b_{ \pm \ell} \sim \mathcal{O}\left(\epsilon^{2^{\ell}}\right)$. Near the $k \approx \omega$ poles, where $k= \pm \omega+\mathcal{O}\left(\epsilon m_{a}\right)$, a factor of $\left(k^{2}-\omega^{2}\right) \propto \epsilon^{-1}$ modifies the power counting to $b_{ \pm \ell} \sim$ $\mathcal{O}\left(\epsilon^{2^{\ell}-1}\right)$.

However, when $\omega \approx \frac{n}{2} m_{a}$ for integer $n$, the power counting is overturned near the $k^{2}=\omega^{2}$ poles, prompting the special treatment in Sec. III A. For example, for $\omega=\frac{1}{2} m_{a} \pm \mathcal{O}\left(\epsilon m_{a}\right)$, the poles in $a_{0}(\omega)$ and $a_{0}\left(\omega-m_{a}\right)$ are encountered simultaneously, and rather than finding
$a_{1}(\omega)=a_{0}(\omega)(1+\mathcal{O}(\epsilon))$, the difference between $a_{1}(\omega)$ and $a_{0}(\omega)$ becomes $\mathcal{O}\left(\epsilon^{0}\right)$; similarly, $b_{-1}\left(\omega \approx m_{a} / 2\right) \sim$ $\mathcal{O}\left(\epsilon^{0}\right)$. Excepting this $\omega= \pm \frac{1}{2} m_{a}$ resonance and the family of higher-order, narrower resonances, the Green function can otherwise be approximated to arbitrary order in $\epsilon$ by

$$
\tilde{g}_{ \pm}(\omega, k)=a_{\ell}(\omega, k)+\mathcal{O}\left(\epsilon^{2^{\ell}-1}\right) .
$$

The $\ell=0$ case is simply the Maxwell theory $\epsilon \rightarrow 0$ result. At $\ell=2$, the expression for the retarded Green function at $\mathcal{O}\left(\epsilon^{2}\right)$ is 


$$
\begin{aligned}
g_{ \pm}\left(t \mid t_{0}, z\right)= & \left(\frac{1}{2} \Theta(z) \Theta\left(t-t_{0}-z\right)+\frac{1}{2} \Theta(-z) \Theta\left(t-t_{0}+z\right)\right)\left(1+\epsilon 4 i \cos \left(\frac{m_{a}\left(t+t_{0}\right)}{2}\right) \sin \left(\frac{m_{a} z}{2}\right)\right. \\
& +\epsilon^{2}\left[-4+4 \cos \left(m_{a}\left(t+t_{0}\right)\right) \cos \left(m_{a} z\right)+2 m_{a}\left(t-t_{0}\right) \cos \left(\frac{m_{a} z}{2}\right) \sin \left(\frac{m_{a}\left(t-t_{0}\right)}{2}\right)\right. \\
& \left.\left.-2 \cos \left(\frac{m_{a}\left(t-t_{0}\right)}{2}\right)\left(\left[-2+2 \cos \left(m_{a}\left(t+t_{0}\right)\right)\right] \cos \left(\frac{m_{a} z}{2}\right)+m_{a} z \sin \left(\frac{m_{a} z}{2}\right)\right)\right]+\mathcal{O}\left(\epsilon^{3}\right)\right),
\end{aligned}
$$

which agrees at $\mathcal{O}(\epsilon)$ and even at $\mathcal{O}\left(\epsilon^{2} m_{a} T\right)$ and $\mathcal{O}\left(\epsilon^{2} m_{a} z\right)$ with the late-time expression Eq. (65). Note that the series expansions of the Bessel $I_{\nu}(z)$ functions are

$$
I_{0}(\sqrt{z})=1+\frac{z}{4}+\frac{z^{2}}{64}+\frac{z^{3}}{2304}+\ldots, \quad \frac{2}{\sqrt{z}} I_{1}(\sqrt{z})=1+\frac{z}{8}+\frac{z^{2}}{192}+\frac{z^{3}}{9216}+\ldots
$$

To express $g_{ \pm}$in terms of $\dot{\theta}_{0}$, recall that a \pm sign is incorporated into the definition of $\epsilon$, where

$$
g_{+}\left(t \mid t_{0}, z\right): \epsilon \rightarrow+\frac{\dot{\theta}_{0}}{4}, \quad g_{-}\left(t \mid t_{0}, z\right): \epsilon \rightarrow-\frac{\dot{\theta}_{0}}{4} .
$$

As a result, the replacement $\epsilon \rightarrow-\epsilon$ is equivalent to switching $g_{+} \leftrightarrow g_{-}$; taking the complex conjugate, $g_{ \pm}^{\star}=g_{\mp}$; or applying the parity transformation $z \rightarrow-z$.

In Appendix A we provide the $\mathcal{O}\left(\epsilon^{3}\right)$ form of $g\left(t \mid t_{0}, z\right)$. We also demonstrate that as $m_{a} T \gg 1$ and $m_{a} z \gg 1$ approach the late-time limit, the leading $\epsilon\left(\epsilon m_{a} T\right)^{n}$ and $\epsilon\left(\epsilon m_{a} z\right)^{n}$ terms reconstruct the series expansions of $I_{0}(\sqrt{\lambda})$ and $I_{1}(\sqrt{\lambda}) / \sqrt{\lambda}$. In Eq. (A2) we verify this explicitly as far as the $1 / 192$ coefficient of the $\epsilon\left(\epsilon m_{a} T\right)^{5}$ term. This indicates that the continued fraction expression for the Green function provides a smooth interpolation between the $\epsilon m_{a} T \ll 1$ and $\epsilon m_{a} T \gg 1$ limits.

By including the $\mathcal{O}\left(\epsilon^{2}\right)$ terms, Eq. (79) is more precise than Eq. (78) in the $\epsilon m_{a} t \ll 1$ limit, and it includes a novel effect: the $-4 \epsilon^{2}$ term in the expansion, which is not proportional to any sinusoidal factors. When the Green function is convolved with a signal of some duration $T$ and some spectrum of frequencies $\Omega$, in the $T \gg m_{a}^{-1}$ limit the sinusoidal terms act as approximate Dirac $\delta$ functions to enhance the modes with $\Omega \approx \frac{n}{2} m_{a}$ for integers $n \geq 1$.

If the axion mass is heavy enough that $m_{a}$ coincides with observable frequencies of light, then this resonant enhancement may be the most easily visible effect. However, for very light ALP dark matter where $m_{a} \sim \omega<2 \pi \cdot \mathcal{O}(\mathrm{kHz})$ corresponds to difficult-to-detect radio waves, the frequency-independent perturbation to the Green function becomes much more significant. As $\epsilon^{2}$ is proportional to $\rho_{a}$, changes in the axion density can modify the strength of visible light passing through it, causing "nongravitational microlensing."

Depending on the cosmological history, some fraction of the axions can clump together to form minihalos with density perturbations $\delta \rho_{a} / \rho_{a}$ potentially much larger than $\mathcal{O}(1)$ [50]. (For recent work, see [51-55].) In addition to the gravitational microlensing, the direct effect from axion electrodynamics on starlight passing through an axion cluster may be detectable if the average value of $\epsilon^{2}=$ $\frac{1}{16} g_{a \gamma \gamma}^{2} \rho_{a} / m_{a}^{2}$ is not too small.

\section{Numeric results}

In Secs. III A and III B we used different methods to approximate the Green function in the homogeneous, oscillating axion background. In this section we verify Eqs. (78) and (79) by comparing them to the numeric solution of the differential equation

$$
\begin{aligned}
& \left(\partial_{t}^{2}-\partial_{z}^{2}+4 i m_{a} \epsilon \cos \left(m_{a} t\right) \partial_{z}\right) g_{ \pm}\left(t-t_{0}, z-z_{0}\right) \\
& \quad=\lim _{\sigma \rightarrow 0} \frac{1}{2 \pi \sigma^{2}} \exp \left(\frac{-\left(t-t_{0}\right)^{2}}{2 \sigma^{2}}\right) \exp \left(\frac{-\left(z-z_{0}\right)^{2}}{2 \sigma^{2}}\right) .
\end{aligned}
$$

(Recall that $\epsilon \equiv \pm \frac{\dot{\theta}_{0}}{4 m_{a}}$.) For the numeric calculations, we use a small value of $\sigma<\epsilon m_{a}^{-1}$ to approximate the delta function source.

In Fig. 5, we compare the series expansion of the continued fraction $\tilde{g}_{ \pm}(\omega, k)=a_{\ell}(\omega, k)$ at $\ell=2$ to the numeric result calculated from Eq. (76) with $\sigma=0.05 m_{a}^{-1}$. Rather than truncating the series expansion at $\mathcal{O}\left(\epsilon^{2}\right)$ as in Eq. (73), we use the $\mathcal{O}\left(\epsilon^{3}\right)$ expression Eq. (A1) from Appendix A. For simplicity, we use $t_{0}=0$ in this example, with $\epsilon=1 / 16$. The agreement at early times, $\epsilon m_{a} t \ll 1$, is quite good, but the approximation begins to fail by $t \gtrsim 2 m_{a}^{-1} / \epsilon$. Even though the $\mathcal{O}\left(\epsilon^{3}\right)$ expression includes terms that grow as $\epsilon\left(\epsilon m_{a} T\right)$ and $i \epsilon\left(\epsilon m_{a} T\right)^{2}$, by $t \geq 45 m_{a}^{-1}$ the exponential growth has begun to invalidate the series expansion in $\left(\epsilon m_{a} T\right)$ for both the real and imaginary parts of the Green function.

Figure 6 shows the superior agreement between the latetime expression Eq. (65) and the numeric result at later times $\epsilon m_{a} t \sim \mathcal{O}(1)$. Compared to the continued fraction solution Eq. (A1), Eq. (65) is missing the $\mathcal{O}\left(\epsilon^{2}\left(\epsilon m_{a} T\right)^{0}\right)$ and $\mathcal{O}\left(\epsilon^{3}\left(\epsilon m_{a} T\right)^{1}\right)$ terms, making it less precise at early times. However, because Eq. (65) includes terms of 

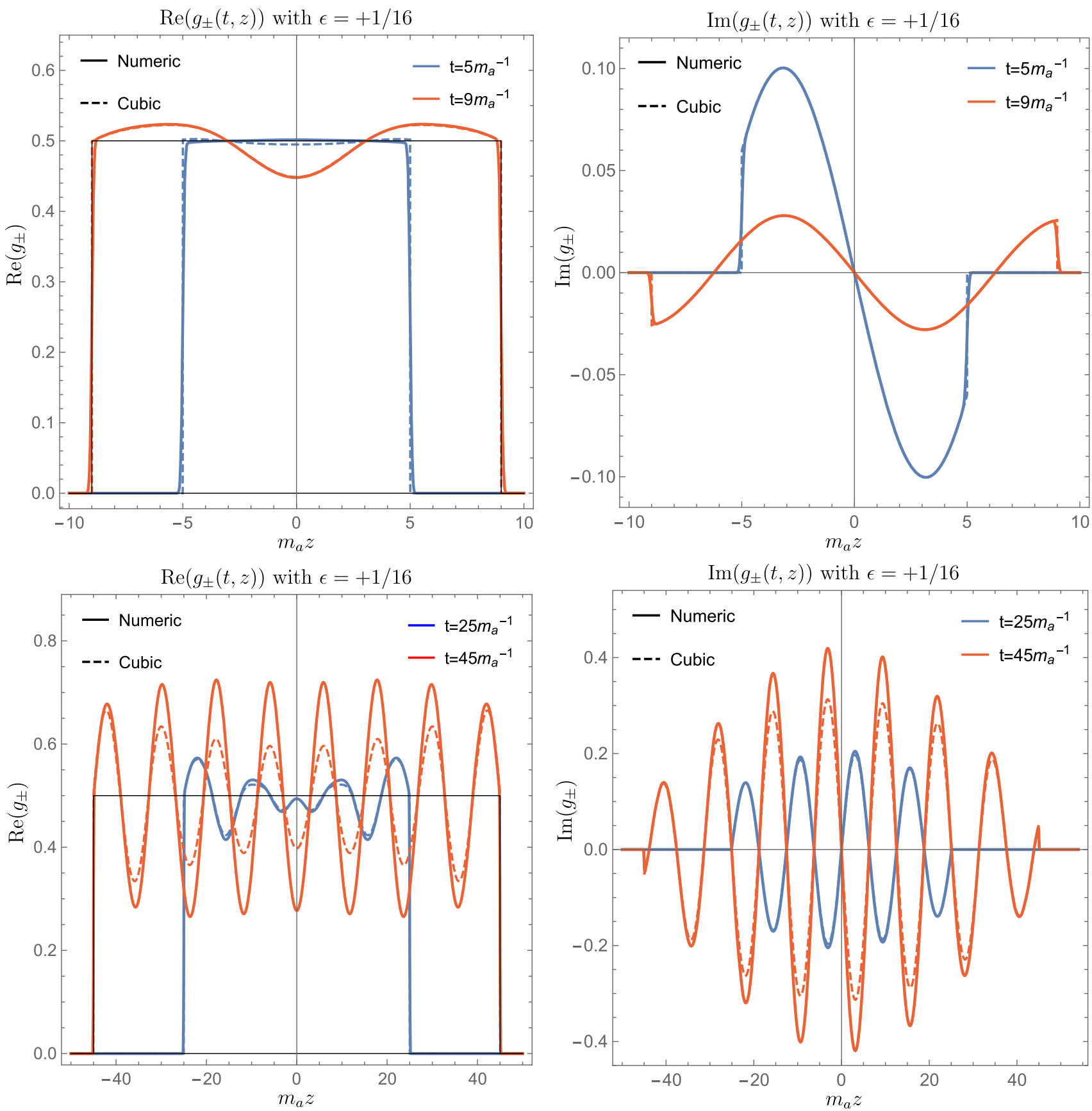

FIG. 5. Left column: the real part of the Green function $\operatorname{Re}\left(g_{ \pm}\left(t \mid t_{0}, z\right)\right)$ is plotted as a function of $z$, comparing the series expansion Eq. (A1) at $\mathcal{O}\left(\epsilon^{3}\right)$ (dashed) against the numeric result (solid) at the following moments in time: $m_{a} t=\{5,9\}$ (top), $m_{a} t=\{25,45\}$ (bottom). A thin black line shows the $\epsilon \equiv \pm \frac{1}{4} \dot{\theta}_{0} / m_{a} \rightarrow 0$ result at $t=9 m_{a}^{-1}$ and $t=45 m_{a}^{-1}$, in the upper and lower panels (respectively). Right column: the series expansion (dashed) and numeric result (solid) for $\operatorname{Im}\left(g_{ \pm}\left(t \mid t_{0}, z\right)\right.$ ) are shown as functions of $z$ for the same fixed values of $t$ as in the left column, at early times $t=\left\{5 m_{a}^{-1}, 9 m_{a}^{-1}\right\}$ (top) and late times $t=\left\{25 m_{a}^{-1}, 45 m_{a}^{-1}\right\}$ (bottom). In the $\epsilon \rightarrow 0$ limit (ordinary Maxwell theory), $\operatorname{Im}\left(g_{ \pm}\right) \rightarrow 0$. Changing the sign of $\epsilon$ is equivalent to replacing $g_{ \pm}$with its complex conjugate, $g_{ \pm} \rightarrow g_{ \pm}^{\star}$, or performing the parity transformation $z \rightarrow-z$. In this example we take $\epsilon=+1 / 16$ and $t_{0}=0$, so that the top and bottom rows represent "early" and "late" times, with $\epsilon m_{a} t \ll 1$ and $\epsilon m_{a} t \gtrsim 1$, respectively. The $t=45 m_{a}^{-1}$ curve in red shows the breakdown of the series expansion for $\epsilon m_{a} t \gg 1$.

$\mathcal{O}\left(\epsilon\left(\epsilon m_{a} T\right)^{n}\right)$ for all nonnegative integers $n$, it is the correct choice for handling $\mathcal{O}(1)$ values of $\epsilon m_{a} T$.

At extremely late times, when $m_{a} T \gg \epsilon^{-2}$, even our "late-time" approximation from Sec. III A fails to capture the dominant behavior of the Green function. In addition to the $\omega= \pm m_{a} / 2$ poles, the contribution from the next-toleading resonance at $\omega= \pm m_{a}+\mathcal{O}\left(\epsilon^{2} m_{a}\right)$ becomes significant. To calculate the extremely late Green function, the 

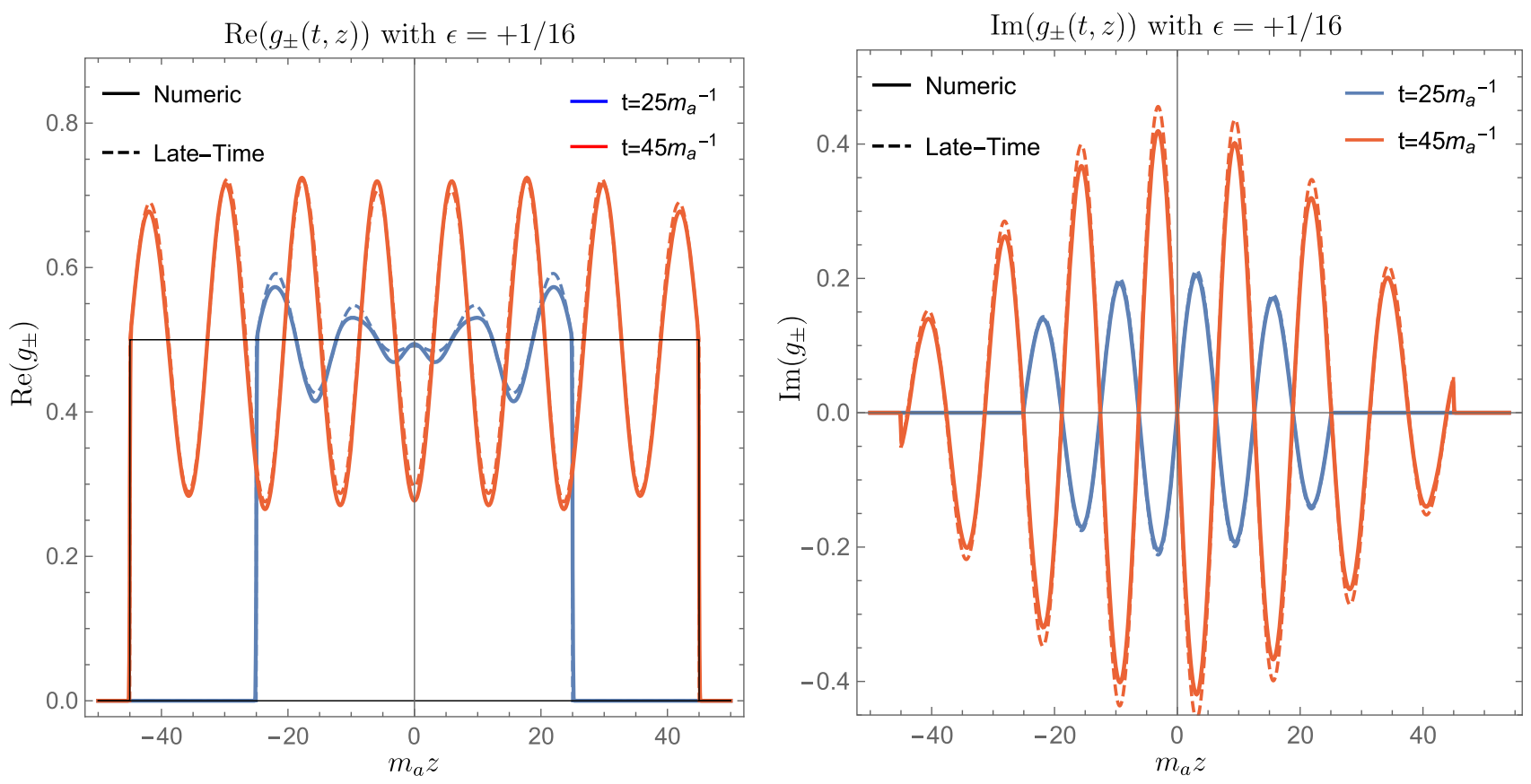

FIG. 6. The real (left) and imaginary (right) parts of the Green function $g\left(t \mid t_{0}, z\right)$ are plotted as functions of $z$ at fixed values of $m_{a} t=\{25,45\}$. In each plot the late-time approximation Eq. (65) (dashed) closely matches the numeric solution (solid), improving on the nonresonant series expansions used in the lower panels of the previous figure. In this example, $\epsilon \equiv \pm \frac{\dot{\theta}_{0}}{4 m_{a}}=+1 / 16$ and $t_{0}=0$, and the numeric calculation was performed with $\sigma=0.05 m_{a}^{-1}$. For reference, the $\epsilon=0$ solution for $\operatorname{Re}\left(g_{ \pm}\right)$is shown at $t=45 m_{a}^{-1}$ (thin black line).

methods of Sec. III A can be repeated with some new $k_{ \pm}=$ $\pm m_{a}+\epsilon^{2} \gamma$ and $\omega_{ \pm}= \pm m_{a}+\epsilon^{2} \delta$. Together with the subleading terms from the $\omega= \pm m_{a} / 2$ poles, the resulting expression would include all terms of $\mathcal{O}\left(\epsilon^{2}\left(m_{a} T\right)^{n}\right)$, and should match the $n=0$ and $n=1$ terms that appear already in Eq. (A1). For the phenomenologically viable values of $\epsilon$ with $\rho_{a} \sim 0.4 \mathrm{GeV} / \mathrm{cm}^{3}$, the decoherence at $T_{c} \sim\left(m_{a} v^{2}\right)^{-1}$ ensures that $\epsilon^{2} m_{a} T \lesssim \mathcal{O}(1)$ for all $T \lesssim T_{c}$, and the axion field loses coherence before the behavior at these "extremely late" times can be explored.

\section{CONCLUSIONS}

In this paper we have computed a set of Green functions appropriate to various limits of axion electrodynamics. Between Secs. II A, III A, and III B, our analysis covers the phenomenologically viable parameter space for coherent axion backgrounds, as well as more extreme ALP models with stronger couplings or enhanced energy densities.

Our study of photon propagation at early times $T \ll m_{a}^{-1}$ in Sec. II shows that the QCD axion induces gentle modifications to the propagation of local disturbances in standard electrodynamics. ALP models in the more extreme corners of parameter space can instigate more dramatic growth in low-frequency modes as signal pulses pass through space. In Sec. III, extending our analysis to account for the oscillation of the axion field, we derive a Green function that preferentially enhances radiation with frequencies close to $\omega=n \frac{m_{a}}{2}$ for positive integers $n$. In all cases, perhaps the sharpest qualitative distinction between radiation in Maxwell theory and in axion electrodynamics is the presence of inside-the-light-cone propagation in the latter.

We collect our main results below. For plane waves symmetric in the $x$ and $y$ directions, the Green functions $g_{ \pm}$satisfy the equations of motion $\left(\partial_{t}^{2}-\partial_{z}^{2} \pm i \dot{\theta}(t) \partial_{z}\right) \times$ $g_{ \pm}\left(t-t_{0}, z-z_{0}\right)=\delta\left(z-z_{0}\right) \delta\left(t-t_{0}\right)$ for right- and leftpolarized light, respectively.

(i) Semistatic limit: at times $t \ll m_{a}^{-1}$, with $\mu=$ $\frac{1}{2} \dot{\theta}_{0} \cos \left(m_{a} t\right) \simeq$ const.:

$$
\begin{aligned}
g_{ \pm}\left(t-t_{0}, z\right)= & e^{ \pm i \mu z} \frac{\Theta\left(t-t_{0}\right) \Theta\left(\left(t-t_{0}\right)^{2}-z^{2}\right)}{2} \\
& \times I_{0}\left(\sqrt{\mu^{2}\left(\left(t-t_{0}\right)^{2}-z^{2}\right)}\right) .
\end{aligned}
$$

The phase factor $e^{ \pm i \mu z}$ provides polarizationdependent phase velocities, as anticipated by the dispersion relation Eq. (13). The exponentially growing $I_{0}$ function corresponds to superluminal group velocities for both polarizations of light. For nonstandard ALP models with $m_{a} \ll \dot{\theta}_{0}$, the exponential growth at $t \gtrsim \mu^{-1}$ can begin while the semistatic condition $t \ll m_{a}^{-1}$ is still satisfied. Otherwise, when $\mu t \lesssim 1$, the Bessel function is well approximated by its series expansion in powers of $\mu t$ and $\mu z$. 
(ii) Resonant, late-time limit: for $|\epsilon| m_{a} t \gtrsim 1$, where $\epsilon m_{a}= \pm \frac{1}{4} \dot{\theta}_{0}$ for $g_{ \pm}$, respectively:

$$
\begin{aligned}
g_{ \pm}\left(t \mid t_{0}, z\right)= & \frac{\Theta\left(t-t_{0}\right) \Theta\left(\left(t-t_{0}\right)^{2}-z^{2}\right)}{2}\left(1+4 i \epsilon I_{0}(\sqrt{\lambda}) \cos \left(\frac{m_{a}\left(t+t_{0}\right)}{2}\right) \sin \left(\frac{m_{a} z}{2}\right)\right. \\
& +4 \epsilon \frac{I_{1}(\sqrt{\lambda})}{\sqrt{\lambda}}\left[m_{a} \epsilon\left(t-t_{0}\right) \sin \left(\frac{m_{a}\left(t-t_{0}\right)}{2}\right) \cos \left(\frac{m_{a} z}{2}\right)\right. \\
& \left.\left.-m_{a} \epsilon z \cos \left(\frac{m_{a}\left(t-t_{0}\right)}{2}\right) \sin \left(\frac{m_{a} z}{2}\right)\right]\right),
\end{aligned}
$$

where $g_{ \pm}$is expressed as a function of $\lambda=\epsilon^{2} m_{a}^{2}\left(\left(t-t_{0}\right)^{2}-z^{2}\right)$. This Green function describes the behavior at late times, $\lambda \gtrsim 1$, when the resonant enhancement of frequencies $\omega \simeq m_{a} / 2$ is the dominant effect. In contrast to Eq. (77), Eq. (78) is appropriate for the standard ALP models with $\dot{\theta}_{0} \ll m_{a}$, and is valid even for $t \gg m_{a}^{-1}$. Both Eqs. (77) and (78) exhibit exponential growth at late times $t \gg \dot{\theta}^{-1}$, though in the latter case the enhancement is specific to frequency modes within the narrow resonance $\omega=m_{a} / 2 \pm \mathcal{O}(\dot{\theta})$.

At extremely late times, when $\epsilon^{2} m_{a} t \gg 1$, the contributions from the higher resonances such as $\omega=m_{a} \pm \mathcal{O}\left(\dot{\theta}^{2} / m_{a}\right)$ also become significant. Given the small values of $\epsilon$ for most regions of ALP parameter space, and the fact that the axion background usually exhibits decoherence before $\epsilon^{2} m_{a} t \sim \mathcal{O}(1)$ is satisfied, we do not provide the Green function in this limit. However, the methods of Sec. III A can be extended in a straightforward manner to describe the dominant behavior in the $\epsilon^{2} m_{a} t \gtrsim 1$ limit.

(iii) Nonresonant propagation: for $|\epsilon| m_{a} t \lesssim 1$, and for signals that do not include the resonant frequencies $\omega=m_{a} / 2+\mathcal{O}\left(\dot{\theta}_{0}\right)$ :

$$
\begin{aligned}
g_{ \pm}\left(t \mid t_{0}, z\right)= & \frac{\Theta\left(t-t_{0}\right) \Theta\left(\left(t-t_{0}\right)^{2}-z^{2}\right)}{2}\left(1+\epsilon 4 i \cos \left(\frac{m_{a}\left(t+t_{0}\right)}{2}\right) \sin \left(\frac{m_{a} z}{2}\right)-4 \epsilon^{2}\right. \\
& +\epsilon^{2}\left[4 \cos \left(m_{a}\left(t+t_{0}\right)\right) \cos \left(m_{a} z\right)+2 m_{a}\left(t-t_{0}\right) \cos \left(\frac{m_{a} z}{2}\right) \sin \left(\frac{m_{a}\left(t-t_{0}\right)}{2}\right)\right. \\
& \left.-2 \cos \left(\frac{m_{a}\left(t-t_{0}\right)}{2}\right)\left(\left[-2+2 \cos \left(m_{a}\left(t+t_{0}\right)\right)\right] \cos \left(\frac{m_{a} z}{2}\right)+m_{a} z \sin \left(\frac{m_{a} z}{2}\right)\right)\right] \\
& \left.+\mathcal{O}\left(\epsilon^{3}\right)\right),
\end{aligned}
$$

where the $\mathcal{O}\left(\epsilon^{3}\right)$ term of the nonresonant series expansion is provided in Eq. (A1) in Appendix A. As long as $|\epsilon| m_{a} t<1$ and $\epsilon \lesssim 1$, the Green function can be calculated to arbitrary precision in $\mathcal{O}\left(\epsilon^{n}\right)$ using this approach. For $|\epsilon| m_{a} t \gtrsim 1$, this series expansion interpolates smoothly onto the Bessel functions in Eq. (65), which include all terms of order $\epsilon\left(\epsilon m_{a} t\right)^{n}$ and $\epsilon\left(\epsilon m_{a} z\right)^{n}$ for $n=0,1,2 \ldots$

In addition to the enhancement of specific frequencies $\omega \approx \frac{n}{2} m_{a}$, Eqs. (73) and (A1) include terms that are not sinusoidal, and which modify the propagation of all frequencies of light. For ALP models with small axion masses, where the resonant frequencies themselves are too small to detect, the frequency independent $\left(1-4 \epsilon^{2}\right)$ part of the Green function continues to affect visible wavelengths of light, and may provide a new signal of ALP dark matter in regions where the axion density $\rho_{a} \propto \epsilon^{2}$ varies significantly.

Both analyses in Sec. III are predicated upon $\epsilon=$ $\pm \frac{1}{4} \dot{\theta}_{0} / m_{a}$ being a small parameter, and so the $\epsilon>\mathcal{O}(1)$ case is generally the most difficult to address. If $\epsilon>1$ then the continued fraction expression for the Green function does not converge. In terms of the resonant analysis in Sec. III A, once $\epsilon^{n} m_{a} t \gtrsim 1$, the Green function receives contributions from an infinite set of poles at $\omega_{n}=\frac{n}{2} m_{a}$. This unusual $m_{a} \ll \dot{\theta}$ limit is handled by the treatment in Section II A for times $t \ll m_{a}^{-1}$, where the semistatic approximation is valid. However, once $t \gg m_{a}^{-1}$, the $\epsilon>1$ Green function must be written in terms of Mathieu functions or calculated numerically. 
If axions make up some component of the dark matter, then their presence may be discerned through their influence on propagating photons. Sensitive terrestrial experiments designed to measure the inside-the-light-cone propagation or the polarization-dependent perturbations from axion electrodynamics might complement existing detection strategies for ALP dark matter. Astrophysical observations may also be sensitive to the effects of axions. Depending on the axion mass, the resonant enhancement and frequency-independent modifications could provide additional opportunities to detect axion dark matter, especially if some fraction of the dark matter has collapsed into minihalos.

The work presented here can be developed in several directions. To fully understand the propagation of light through a galaxy containing axions, especially in the neighborhood of axion minihalos or other dense objects, it is important to consider the effects from spatial gradients in the axion background. For light propagating through multiple coherent patches, over distances $L \gtrsim\left(m_{a} v\right)^{-1}$ or for times $T \gtrsim\left(m_{a} v^{2}\right)^{-1}$, decoherence effects are similarly important. In physical systems where the axion density is enhanced, or where its coherence time is increased, the axion-induced effects on propagating light become more significant. Possible applications include minihalos, cold streams and caustics in the galaxy, as well as much denser structures such as axion strings, or domain walls in the early Universe. Finally, Green function techniques may provide useful tools for exploring the sensitivity of terrestrial axion detection experiments. We hope to explore these possibilities in future work.

\section{ACKNOWLEDGMENTS}

We are grateful to Carlos Blanco, Nico Fernandez and Matt Reece for helpful conversations, and to Yoni Kahn for collaboration in the early stages of the work. The work of P. D. and B. L. is supported in part by the National Science Foundation Grant No. PHY-1719642, and the work of P. A., P. D. and B.L. is supported in part by the US Department of Energy Grant No. DE-SC0015655. P. A. acknowledges the hospitality of the Kavli Institute for Theoretical Physics, which is supported in part by the National Science Foundation under Grant No. NSF-PHY1748958. B. L. thanks the Simons Center for Geometry and Physics, Stony Brook University at which some of the research for this paper was performed.

\section{APPENDIX A: CONTINUED FRACTION GREEN FUNCTION AT HIGHER ORDER}

In Sec. III B, we listed the first few terms of the continued fraction Eq. (71), truncating the series expansion of $a_{2}(\omega, k)$ at $\mathcal{O}\left(\epsilon^{2}\right)$. The number of terms in the expansion increases rapidly for higher powers of $\epsilon$. For example, the $\mathcal{O}\left(\epsilon^{3}\right)$ expression for the Green function is

$$
\begin{aligned}
g\left(t \mid t_{0}, z\right)= & \frac{\Theta\left(t-t_{0}\right) \Theta\left(\left(t-t_{0}\right)^{2}-z^{2}\right)}{2}\left(1+\epsilon 4 i \cos \left(\frac{m_{a}\left(t+t_{0}\right)}{2}\right) \sin \left(\frac{m_{a} z}{2}\right)\right. \\
& +\epsilon^{2}\left[-4+4 \cos \left(m_{a}\left(t+t_{0}\right)\right) \cos \left(m_{a} z\right)+2 m_{a}\left(t-t_{0}\right) \cos \left(\frac{m_{a} z}{2}\right) \sin \left(\frac{m_{a}\left(t-t_{0}\right)}{2}\right)\right. \\
& \left.-2 \cos \left(\frac{m_{a}\left(t-t_{0}\right)}{2}\right)\left(\left[-2+2 \cos \left(m_{a}\left(t+t_{0}\right)\right)\right] \cos \left(\frac{m_{a} z}{2}\right)+m_{a} z \sin \left(\frac{m_{a} z}{2}\right)\right)\right] \\
& +\frac{i}{3} \epsilon^{3}\left\{\operatorname { c o s } ( \frac { m _ { a } ( t + t _ { 0 } ) } { 2 } ) \left(12 m_{a} z \cos \frac{m_{a} z}{2} \sin ^{2}\left(\frac{m_{a}\left(t-t_{0}\right)}{2}\right)\right.\right. \\
& +\sin \left(\frac{m_{a} z}{2}\right)\left[2 \cos \left(2 m_{a} t\right)-38 \cos \left(m_{a}\left(t-t_{0}\right)\right)+2 \cos \left(2 m_{a} t_{0}\right)+6 \cos \left(m_{a}\left(t+t_{0}\right)\right)\right. \\
& \left.\left.-63-6 m_{a}\left(t-t_{0}\right) \sin \left(m_{a}\left(t-t_{0}\right)\right)+3 m_{a}^{2}\left(t-t_{0}\right)^{2}-3 m_{a}^{2} z^{2}\right]\right) \\
& +8 \sin \left(m_{a} z\right)\left[3 \cos \left(m_{a} t\right)+3 \cos \left(m_{a} t_{0}\right)-\cos \left(m_{a}\left(2 t+t_{0}\right)\right)-\cos \left(m_{a}\left(t+2 t_{0}\right)\right)\right] \\
& \left.\left.+9 \cos \left(\frac{3 m_{a}\left(t+t_{0}\right)}{2}\right) \sin \left(\frac{3 m_{a} z}{2}\right)\right\}+\mathcal{O}\left(\epsilon^{4}\right)\right) .
\end{aligned}
$$

To calculate $g_{ \pm}\left(t \mid t_{0}, z\right)$ to $\mathcal{O}\left(\epsilon^{4}\right)-\mathcal{O}\left(\epsilon^{7}\right)$, it becomes necessary to integrate the series expansion of $a_{\ell=3}(\omega, k)$ rather than $a_{2}(\omega, k)$, which can become tedious. At $\mathcal{O}\left(\epsilon^{4}\right)$, for example, $a_{3}(\omega, k)$ includes triple poles $\left(k^{2}-\omega^{2}\right)^{3}$, double poles $\left(k^{2}-\left(\omega \pm m_{a}\right)^{2}\right)^{2}$ and $\left(k^{2}-\left(\omega \pm 2 m_{a}\right)^{2}\right)^{2}$ and single poles $\left(k^{2}-\left(\omega \pm 3 m_{a}\right)^{2}\right)$ and $\left(k^{2}-\left(\omega \pm 4 m_{a}\right)^{2}\right)$. After integrating over $k$, the $\mathcal{O}\left(\epsilon^{4}\right)$ component yields quadruple poles at $\omega= \pm \frac{1}{2} m_{a}$, triple poles at $\omega= \pm \frac{3}{2} m_{a}$, double poles at $\omega= \pm m_{a}$ and $\omega= \pm \frac{5}{2} m_{a}$, and single poles at $\omega=0, \omega= \pm 2 m_{a}, \omega= \pm 3 m_{a}$ and $\omega= \pm \frac{7}{2} m_{a}$. 
Already at $\mathcal{O}\left(\epsilon^{3}\right)$, we can see that the $\mathcal{O}\left(\epsilon\left(\epsilon m_{a} T\right)^{n}\right)$ terms for $n=0,1,2$ match the series expansions of the Bessel functions $I_{0}$ and $I_{1}$ given in Eq. (74), an important consistency check between Secs. III A and III B. By focusing exclusively on the poles at $k^{2}=\omega^{2}$ and $k^{2}=\left(\omega \pm m_{a}\right)^{2}$, we can verify Eq. (65) by comparing it to the $\mathcal{O}\left(\epsilon\left(\epsilon m_{a} T\right)^{n}\right)$ expansion for arbitrarily large $n$. For example, at $n=5$, we integrate the $\mathcal{O}\left(\epsilon^{6}\right)$ expression for $a_{3}(\omega, k)$ to find that

$$
\begin{aligned}
g\left(t \mid t_{0}, z\right)= & \left(\frac{1}{2} \Theta(z) \Theta\left(t-t_{0}-z\right)+\frac{1}{2} \Theta(-z) \Theta\left(t-t_{0}+z\right)\right)\left(1+4 i \epsilon \cos \left(\frac{m_{a}\left(t+t_{0}\right)}{2}\right) \sin \left(\frac{m_{a} z}{2}\right)\right. \\
& \times\left(1+\frac{\epsilon^{2}}{4}\left(m_{a}^{2}\left(t-t_{0}\right)^{2}-m_{a}^{2} z^{2}\right)+\frac{\epsilon^{4}}{64}\left(m_{a}^{2}\left(t-t_{0}\right)^{2}-m_{a}^{2} z^{2}\right)^{2}+\ldots\right) \\
& +2 \epsilon^{2}\left(m_{a}\left(t-t_{0}\right) \cos \left(\frac{m_{a} z}{2}\right) \sin \left(\frac{m_{a}\left(t-t_{0}\right)}{2}\right)-m_{a} z \sin \left(\frac{m_{a} z}{2}\right) \cos \left(\frac{m_{a}\left(t-t_{0}\right)}{2}\right)\right) \\
& \left.\times\left(1+\frac{\epsilon^{2}}{8}\left(m_{a}^{2}\left(t-t_{0}\right)^{2}-m_{a}^{2} z^{2}\right)+\frac{\epsilon^{4}}{192}\left(m_{a}^{2}\left(t-t_{0}\right)^{2}-m_{a}^{2} z^{2}\right)^{2}+\ldots\right)\right) \\
& +\mathcal{O}\left(\epsilon^{2}\left(\epsilon m_{a} T\right)^{n}\right),
\end{aligned}
$$

in complete agreement with Eq. (65).

\section{APPENDIX B: INTEGRAL FOR FOUR-DIMENSIONAL GREEN FUNCTION}

The part of the Green function $G_{i j}$ with the $k_{i} k_{j} / k^{2}$ tensor structure cannot be easily expressed in terms of hypergeometric functions. The problematic integral can be expressed as

$$
A_{i j}=\frac{1}{4 \pi} \partial_{i} \partial_{j}\left[\frac{1}{2} \Theta(t) \Theta\left(t^{2}-r^{2}\right) \frac{2}{\mu r} \mathcal{I}(t, r)\right]
$$

where

$$
\mathcal{I}=\frac{\mu r}{2} \int_{-1}^{1} d q \cos (q r \mu) I_{0}\left(\sqrt{\mu^{2} t^{2}-\mu^{2} q^{2} r^{2}}\right)=\int_{0}^{\mu r} d(q \mu r) \cos (q r \mu) I_{0}\left(\sqrt{(\mu t)^{2}-(\mu q r)^{2}}\right) .
$$

Defining the dimensionless parameters

$$
v \equiv \frac{1}{4} q^{2} \mu^{2} r^{2}, \quad v_{0} \equiv \frac{1}{4} \mu^{2} r^{2}, \quad \sigma \equiv \frac{1}{4} \mu^{2} t^{2},
$$

and replacing the trigonometric and Bessel functions with their equivalent hypergeometric functions,

$$
\begin{aligned}
& \mathcal{I}=\int_{0}^{v_{0}} \frac{d v}{\sqrt{v}}{ }_{0} F_{1}\left(\begin{array}{c}
- \\
\frac{1}{2}
\end{array} \mid-v\right){ }_{0} F_{1}\left(\begin{array}{c}
- \\
1
\end{array} \mid \sigma-v\right)=\int_{0}^{v_{0}} \frac{d v}{\sqrt{v}}{ }_{0} F_{1}\left(\begin{array}{c}
- \\
\frac{1}{2}
\end{array} \mid-v\right) \sum_{k=0}^{\infty} \frac{(\sigma-v)^{k}}{(k !)^{2}}, \\
& =\int_{0}^{v_{0}} \frac{d v}{\sqrt{v}}{ }_{0} F_{1}\left(\begin{array}{c}
- \\
\frac{1}{2}
\end{array} \mid-v\right) \sum_{k=0}^{\infty} \sum_{j=0}^{k} \frac{\sigma^{k-j}(-v)^{j}}{k ! j !(k-j) !},
\end{aligned}
$$

the integral over $v$ can be completed:

$$
\int_{0}^{v_{0}} d v v^{j-\frac{1}{2}} F_{1}\left(\begin{array}{c}
- \\
\frac{1}{2}
\end{array} \mid-v\right)=\frac{v_{0}^{j+\frac{1}{2}}}{j+\frac{1}{2}} 1 F_{2}\left(\begin{array}{c}
j+\frac{1}{2} \\
\frac{1}{2}, \frac{j}{2}+\frac{1}{4}
\end{array} \mid-v_{0}\right),
$$

with the result 


$$
\mathcal{I}=\sum_{k=0}^{\infty} \sum_{j=0}^{k} \frac{\sigma^{k-j} v_{0}^{j+\frac{1}{2}}(-1)^{j}}{k ! j !(k-j) !\left(j+\frac{1}{2}\right)}{ }_{1} F_{2}\left(\begin{array}{c}
j+1 \\
\frac{1}{2}, \frac{j}{2}+\frac{1}{4}
\end{array} \mid-v_{0}\right) .
$$

One of the two infinite series can be replaced by a hypergeometric function, after replacing the index $k$ with $\ell \equiv k-j$, so that both $j$ and $\ell$ run from zero to infinity:

$$
\begin{aligned}
& \mathcal{I}=\sqrt{v_{0}} \sum_{j=0}^{\infty} \sum_{\ell=0}^{\infty} \frac{\sigma^{\ell}}{\ell ! \Gamma(j+\ell+1)} \frac{\left(-v_{0}\right)^{j}}{j !\left(j+\frac{1}{2}\right)}{ }_{1} F_{2}\left(\begin{array}{c}
j+\frac{1}{2} \\
\frac{1}{2}, \frac{j}{2}+\frac{1}{4}
\end{array} \mid-v_{0}\right), \\
& \mathcal{I}=\sqrt{v_{0}} \sum_{j=0}^{\infty} \frac{\left(-\frac{1}{4} \mu^{2} r^{2}\right)^{j}}{j !\left(j+\frac{1}{2}\right)}\left(\frac{2}{\mu t}\right)^{j} I_{j}(\mu t)_{1} F_{2}\left(\begin{array}{c}
j+\frac{1}{2} \\
\frac{1}{2}, \frac{j}{2}+\frac{1}{4}
\end{array} \mid-\frac{1}{4} \mu^{2} r^{2}\right) .
\end{aligned}
$$

Even for relatively large values of $\mu t$ and $\mu r$, the series expression for $\mathcal{I}$ converges relatively quickly. In the asymptotic $v_{0} \gg 1$ limit, the ${ }_{1} F_{2}$ function is approximately

$$
\lim _{v_{0} \rightarrow \infty} F_{2}\left(\begin{array}{c}
j+\frac{1}{2} \\
\frac{1}{2}, \frac{j}{2}+\frac{1}{4}
\end{array} \mid-v_{0}\right) \approx v_{0}^{j / 4+1 / 8} \frac{2^{-j+1 / 2} \sqrt{\pi}}{\Gamma(3 / 4+j / 2)} \cos \left(2 \sqrt{v_{0}}+\frac{\pi(1+2 j)}{8}\right),
$$

while to leading order the Bessel functions $I_{j}(2 \sqrt{\sigma})$ approach

$$
\lim _{\mu t \rightarrow \infty} I_{j}(\mu t) \approx \frac{e^{\mu t}}{\sqrt{2 \pi \mu t}}\left(1-\frac{\left(4 j^{2}-1\right)}{8 \mu t}+\mathcal{O}\left((\mu t)^{-2}\right)\right)
$$

Consequently, the number of terms in $\sum_{j=0}^{j_{\max }}$ required for convergence is driven primarily by the value of $\mu t$ : in the limit $\mu t \gg 1$, the series converges quickly for $j>j_{\max }$ once

$$
\frac{3 j_{\max }}{2} \log \frac{j_{\max }}{e}+j_{\max } \log \frac{\mu t}{\sqrt{2}}-\frac{5 j_{\max }}{2} \log \frac{\mu r}{2} \gg \mu t .
$$

[1] R. Peccei and H. R. Quinn, $C P$ Conservation in the Presence of Instantons, Phys. Rev. Lett. 38, 1440 (1977).

[2] R. D. Peccei and H. R. Quinn, Constraints imposed by $C P$ conservation in the presence of instantons, Phys. Rev. D 16, 1791 (1977).

[3] C. A. Baker et al., An Improved Experimental Limit on the Electric Dipole Moment of the Neutron, Phys. Rev. Lett. 97, 131801 (2006).

[4] J. M. Pendlebury et al., Revised experimental upper limit on the electric dipole moment of the neutron, Phys. Rev. D 92, 092003 (2015).

[5] L. F. Abbott and P. Sikivie, A cosmological bound on the invisible axion, Phys. Lett. 120B, 133 (1983).

[6] J. Preskill, M. B. Wise, and F. Wilczek, Cosmology of the invisible axion, Phys. Lett. 120B, 127 (1983).

[7] M. Dine and W. Fischler, The not so harmless axion, Phys. Lett. 120B, 137 (1983).
[8] H. Primakoff, Photoproduction of neutral mesons in nuclear electric fields and the mean life of the neutral meson, Phys. Rev. 81, 899 (1951).

[9] P. Sikivie, Experimental Tests of the Invisible Axion, Phys. Rev. Lett. 51, 1415 (1983).

[10] D. A. Dicus, E. W. Kolb, V. L. Teplitz, and R. V. Wagoner, Astrophysical bounds on very low mass axions, Phys. Rev. D 22, 839 (1980).

[11] G. G. Raffelt, Astrophysical axion bounds, Lect. Notes Phys. 741, 51 (2008).

[12] V. Anastassopoulos et al. (CAST Collaboration), New CAST limit on the axion-photon interaction, Nat. Phys. 13, 584 (2017).

[13] J. W. Brockway, E. D. Carlson, and G. G. Raffelt, SN1987A gamma-ray limits on the conversion of pseudoscalars, Phys. Lett. B 383, 439 (1996).

[14] J. Grifols, E. Masso, and R. Toldra, Gamma-Rays from SN1987A Due to Pseudoscalar Conversion, Phys. Rev. Lett. 77, 2372 (1996). 
[15] J. P. Conlon, F. Day, N. Jennings, S. Krippendorf, and M. Rummel, Constraints on axion-like particles from nonobservation of spectral modulations for x-ray point sources, J. Cosmol. Astropart. Phys. 07 (2017) 005.

[16] N. Du et al. (ADMX Collaboration), A Search for Invisible Axion Dark Matter with the Axion Dark Matter Experiment, Phys. Rev. Lett. 120, 151301 (2018).

[17] J. L. Ouellet et al., First Results from ABRACADABRA$10 \mathrm{~cm}$ : A Search for Sub- $\mu \mathrm{eV}$ Axion Dark Matter, Phys. Rev. Lett. 122, 121802 (2019).

[18] L. Zhong et al. (HAYSTAC Collaboration), Results from phase 1 of the HAYSTAC microwave cavity axion experiment, Phys. Rev. D 97, 092001 (2018).

[19] E. Armengaud et al. (IAXO Collaboration), Physics potential of the International Axion Observatory (IAXO), J. Cosmol. Astropart. Phys. 06 (2019) 047.

[20] A. Berlin, R. T. D’Agnolo, S. A. R. Ellis, C. Nantista, J. Neilson, P. Schuster, S. Tantawi, N. Toro, and K. Zhou, Axion dark matter detection by superconducting resonant frequency conversion, J. High Energy Phys. 07 (2020) 088.

[21] R. Lasenby, Microwave cavity searches for low-frequency axion dark matter, Phys. Rev. D 102, 015008 (2020).

[22] F. Wilczek, Two Applications of Axion Electrodynamics, Phys. Rev. Lett. 58, 1799 (1987).

[23] S. M. Carroll, G. B. Field, and R. Jackiw, Limits on a Lorentz and parity violating modification of electrodynamics, Phys. Rev. D 41, 1231 (1990).

[24] C. Coriano, Electrodynamics in the presence of an axion, Mod. Phys. Lett. A 07, 1253 (1992).

[25] Z. Qiu, G. Cao, and X.-G. Huang, On electrodynamics of chiral matter, Phys. Rev. D 95, 036002 (2017).

[26] D. Blas, A. Caputo, M. M. Ivanov, and L. Sberna, No chiral light bending by clumps of axion-like particles, Phys. Dark Universe 27, 100428 (2020).

[27] J. I. McDonald and L. B. Ventura, Optical properties of dynamical axion backgrounds, Phys. Rev. D 101, 123503 (2020).

[28] D. Yoshida and J. Soda, Electromagnetic waves propagating in the string axiverse, Prog. Theor. Exp. Phys. 2018, 041 E01 (2018).

[29] W. DeRocco and A. Hook, Axion interferometry, Phys. Rev. D 98, 035021 (2018).

[30] I. Obata, T. Fujita, and Y. Michimura, Optical Ring Cavity Search for Axion Dark Matter, Phys. Rev. Lett. 121, 161301 (2018).

[31] L. M. Krauss, Axions and atomic clocks, arXiv:1905.10014.

[32] S. Chigusa, T. Moroi, and K. Nakayama, Signals of axion like dark matter in time dependent polarization of light, Phys. Lett. B 803, 135288 (2020).

[33] M. Huang and P. Sikivie, The structure of axionic domain walls, Phys. Rev. D 32, 1560 (1985).

[34] Y. Aharonov, A. Komar, and L. Susskind, Superluminal behavior, causality, and instability, Phys. Rev. 182, 1400 (1969).

[35] P. Arias, D. Cadamuro, M. Goodsell, J. Jaeckel, J. Redondo, and A. Ringwald, WISPy cold dark matter, J. Cosmol. Astropart. Phys. 06 (2012) 013.

[36] N. Blinov, M. J. Dolan, P. Draper, and J. Kozaczuk, Dark matter targets for axionlike particle searches, Phys. Rev. D 100, 015049 (2019).
[37] L. Di Luzio, M. Giannotti, E. Nardi, and L. Visinelli, The landscape of QCD axion models, Phys. Rep. 870, 1 (2020).

[38] E. Armengaud, N. Palanque-Delabrouille, C. Yche, D. J. E. Marsh, and J. Baur, Constraining the mass of light bosonic dark matter using SDSS Lyman- $\alpha$ forest, Mon. Not. R. Astron. Soc. 471, 4606 (2017).

[39] V. Iri, M. Viel, M. G. Haehnelt, J. S. Bolton, and G. D. Becker, First Constraints on Fuzzy Dark Matter from Lyman- $\alpha$ Forest Data and Hydrodynamical Simulations, Phys. Rev. Lett. 119, 031302 (2017).

[40] T. Kobayashi, R. Murgia, A. De Simone, V. Iri, and M. Viel, Lyman- $\alpha$ constraints on ultralight scalar dark matter: Implications for the early and late universe, Phys. Rev. D 96, 123514 (2017).

[41] M. Nori, R. Murgia, V. Iri, M. Baldi, and M. Viel, Lyman $\alpha$ forest and non-linear structure characterization in fuzzy dark matter cosmologies, Mon. Not. R. Astron. Soc. 482, 3227 (2019).

[42] A. Arvanitaki, S. Dimopoulos, M. Galanis, L. Lehner, J. O. Thompson, and K. Van Tilburg, Large-misalignment mechanism for the formation of compact axion structures: Signatures from the QCD axion to fuzzy dark matter, Phys. Rev. D 101, 083014 (2020).

[43] A. Arza, Photon enhancement in a homogeneous axion dark matter background, Eur. Phys. J. C 79, 250 (2019).

[44] M. P. Hertzberg and E. D. Schiappacasse, Dark matter axion clump resonance of photons, J. Cosmol. Astropart. Phys. 11 (2018) 004.

[45] A. Arza, T. Schwetz, and E. Todarello, How to suppress exponential growth-On the parametric resonance of photons in an axion background, J. Cosmol. Astropart. Phys. 10 (2020) 013.

[46] P. Sikivie, Invisible axion search methods, arXiv:2003 .02206 .

[47] B. P. Abbott et al., Sensitivity of the Advanced LIGO detectors at the beginning of gravitational wave astronomy, Phys. Rev. D 93, 112004 (2016).

[48] B. Abbott et al. (LIGO Scientific Collaboration), LIGO: The laser interferometer gravitational-wave observatory, Rep. Prog. Phys. 72, 076901 (2009).

[49] A. Vanel, R. Craster, D. Colquitt, and M. Makwana, Asymptotics of dynamic lattice Green's functions, Wave Motion 67, 15 (2016).

[50] E. W. Kolb and I. I. Tkachev, Large amplitude isothermal fluctuations and high density dark matter clumps, Phys. Rev. D 50, 769 (1994).

[51] A. E. Nelson and H. Xiao, Axion cosmology with early matter domination, Phys. Rev. D 98, 063516 (2018).

[52] L. Visinelli and J. Redondo, Axion miniclusters in modified cosmological histories, Phys. Rev. D 101, 023008 (2020).

[53] M. Buschmann, J. W. Foster, and B. R. Safdi, EarlyUniverse Simulations of the Cosmological Axion, Phys. Rev. Lett. 124, 161103 (2020).

[54] B. Eggemeier, J. Redondo, K. Dolag, J. C. Niemeyer, and A. Vaquero, First Simulations of Axion Minicluster Halos, Phys. Rev. Lett. 125, 041301 (2020).

[55] N. Blinov, M. J. Dolan, and P. Draper, Imprints of the Early Universe on axion dark matter substructure, Phys. Rev. D 101, 035002 (2020). 